\title{
Muscleblind-like 2 controls the hypoxia response of cancer cells
}

\author{
SANDRA FISCHER, ${ }^{1}$ ANTONELLA DI LIDDO, ${ }^{2}$ KATARZYNA TAYLOR, ${ }^{3}$ JAMINA S. GERHARDUS, ${ }^{1}$ \\ KRZYSZTOF SOBCZAK, ${ }^{3} \mathrm{KATHI}^{2}$ ZARNACK, ${ }^{2}$ and JULIA E. WEIGAND ${ }^{1}$ \\ ${ }^{1}$ Department of Biology, Technical University of Darmstadt, Darmstadt, 64287, Germany \\ ${ }^{2}$ Buchmann Institute for Molecular Life Sciences, Goethe University Frankfurt, Frankfurt am Main, 60438, Germany \\ ${ }^{3}$ Department of Gene Expression, Institute of Molecular Biology and Biotechnology, Faculty of Biology, Adam Mickiewicz University, \\ Poznan, 61-614, Poland
}

\begin{abstract}
Hypoxia is a hallmark of solid cancers, supporting proliferation, angiogenesis, and escape from apoptosis. There is still limited understanding of how cancer cells adapt to hypoxic conditions and survive. We analyzed transcriptome changes of human lung and breast cancer cells under chronic hypoxia. Hypoxia induced highly concordant changes in transcript abundance, but divergent splicing responses, underlining the cell type-specificity of alternative splicing programs. While RNAbinding proteins were predominantly reduced, hypoxia specifically induced muscleblind-like protein 2 (MBNL2). Strikingly, MBNL2 induction was critical for hypoxia adaptation by controlling the transcript abundance of hypoxia response genes, such as vascular endothelial growth factor A (VEGFA). MBNL2 depletion reduced the proliferation and migration of cancer cells, demonstrating an important role of MBNL2 as cancer driver. Hypoxia control is specific for MBNL2 and not shared by its paralog MBNL1. Thus, our study revealed MBNL2 as central mediator of cancer cell responses to hypoxia, regulating the expression and alternative splicing of hypoxia-induced genes.
\end{abstract}

Keywords: hypoxia; cancer; MBNL2; HIF target genes; alternative splicing

\section{INTRODUCTION}

Hypoxia is a common feature of solid tumors as the oxygen supply to the rapidly proliferating cancer cells is inadequate. Two types of tumor hypoxia occur as a result of structurally and functionally abnormal tumor vasculature. "Chronic" hypoxia arises in cancer cells that are too far away from the blood vessels to be supplied with sufficient oxygen by diffusion. "Acute" hypoxia is the result of temporarily limited perfusion by immature tumor vessels (Vaupel et al. 2004). Such hypoxic regions are spatially and temporally dynamic due to tumor angiogenesis and the reversibility of acute hypoxia, resulting in severe intraand intertumoral heterogeneity of cellular oxygen levels. The oxygen content in hypoxic areas of solid tumors is typically $<1.4 \%$ and may reach complete anoxia (Vaupel et al. 2007).

Hypoxia promotes virtually every step in tumor progression contributing to metabolic reprogramming, angiogenesis, extracellular matrix remodeling, epithelial to mesenchymal transition, cancer stem cell maintenance, immune evasion, and cell death resistance (Schito and

Corresponding author: julia.weigand@tu-darmstadt.de

Article is online at http://www.rnajournal.org/cgi/doi/10.1261/rna. 073353.119.
Semenza 2016). Thus, tumor hypoxia signifies a more aggressive phenotype resulting from increased growth and metastasis. Moreover, tumor hypoxia counteracts radio-, chemo-, and immunotherapy, highlighting the importance of developing effective hypoxia-targeted therapies (Graham and Unger 2018). Well studied is the role of the HIF (hypoxia inducible factor) transcription factors in the adaptation to hypoxia. HIFs are heterodimers consisting of an oxygen-labile $\alpha$ subunit and a constitutively stable $\beta$ subunit. Under hypoxia, HIF $\alpha$ subunits are stabilized, allowing for the transcriptional activation of several hundred target genes (Samanta and Semenza 2018).

Independent from transcriptional adaptation, hypoxia triggers a widespread post-transcriptional response by altering alternative splicing (AS) decisions, translation efficiency, and mRNA stability. For example, at low glucose conditions, several mRNAs of HIF target genes are stabilized under hypoxia (Carraway et al. 2017; Fry et al. 2017). While canonical protein synthesis is reduced due

(C) 2020 Fischer et al. This article is distributed exclusively by the RNA Society for the first 12 months after the full-issue publication date (see http://rnajournal.cshlp.org/site/misc/terms.xhtml). After 12 months, it is available under a Creative Commons License (Attribution-NonCommercial 4.0 International), as described at http:// creativecommons.org/licenses/by-nc/4.0/. 
to the limited ATP supply during hypoxia, alternative translation pathways are activated, to sustain translation of proteins essential for hypoxia adaptation (for review, see Chee et al. 2019). Remarkably, ribosomal profiling of hypoxic primary hepatocytes showed that changes in translation even precede the transcriptional response (Hettiarachchi et al. 2019). In addition, hypoxia-driven AS events diversify the cellular response to hypoxia, both in primary and cancerous cells (Weigand et al. 2012; Hu et al. 2014; Brady et al. 2017; Han et al. 2017; Bowler et al. 2018). The current knowledge of the factors that control these post-transcriptional pathways is still very limited, but crucial in understanding the tumor-promoting effects of hypoxia.

In line with the central role of RNA-binding proteins (RBPs) in post-transcriptional gene control, aberrant RBP activities have been identified as potent cancer drivers (for reviews, see Anczuków and Krainer 2016; Pereira et al. 2017). Just recently, MBNL proteins were found to affect tumorigenesis (Perron et al. 2018; Tabaglio et al. 2018). The MBNL family consists of the three closely related paralogs, MBNL1, MBNL2, and MBNL3 (for review, see Konieczny et al. 2014). MBNL1 and MBNL2 are ubiquitously expressed, whereas MBNL3 expression is more restricted, with highest levels found in the placenta (Fardaei et al. 2002). All three MBNL paralogs encode two amino-terminal tandem zinc finger ( $\mathrm{ZnF}$ ) domains. These bind to RNAs containing two or more clustered 5'-YGCY-3' motifs (Lambert et al. 2014). The ability of MBNLs to bind RNA is further altered by the structural context of the RNA targets (deLorimier et al. 2017; Taylor et al. 2018). Binding of MBNLs activates or represses mRNA alternative splicing depending on MBNL binding location (Charizanis et al. 2012; Wang et al. 2012), with MBNL1 being the most potent splicing regulator among the three paralogs (Sznajder et al. 2016). Additionally, MBNL proteins are proposed to regulate mRNA stability (Masuda et al. 2012) and localization (Adereth et al. 2005; Wang et al. 2012), alternative polyadenylation (Batra et al. 2014), and miRNA biogenesis (Rau et al. 2011). All three MBNL proteins undergo autoregulatory feedback loops and can functionally compensate each other in some cases (Konieczny et al. 2018).

In order to identify RBPs central to the post-transcriptional response of cancer cells during hypoxia, we obtained a comparative set of hypoxia-driven changes in gene and isoform expression as well as changes in RBP levels in two cancer types. We focused on lung and breast cancer, since these are the most common cause for cancer-related deaths in males and females, respectively (Bray et al. 2018; Siegel et al. 2019). Our data show highly correlated changes in transcript abundance, but strongly divergent changes in AS. Strikingly, chronic hypoxia specifically induces MBNL2, while overall RBP levels are reduced. We demonstrate a major role of MBNL2 in promoting hypoxia-dependent gene expression changes, both at the level of transcript abundance and AS. Accordingly, knockdown of MBNL2 in cancer cells leads to reduced proliferation and migration, emphasizing MBNL2 as a driver of cancer cell function in hypoxia.

\section{RESULTS}

\section{Chronic hypoxia induces similar changes in transcript abundance, but divergent alternative splicing programs}

We chose A549 lung and MCF-7 breast cancer cells to compare transcriptomic changes in response to chronic hypoxia, as both cell lines have no deletions or mutations within the HIF pathway, the two major families of splicing factors, the SR proteins (serin/arginine rich) and hnRNPs (heterogeneous nuclear ribonucleoproteins) or core spliceosomal genes, such as mutations in SF3B1 (splicing factor 3B1), which are common in cancer (Cerami et al. 2012; Gao et al. 2013; Seiler et al. 2018). First, we identified the lowest oxygen concentration that would allow continued survival under hypoxic stress. We incubated A549 and MCF-7 cells for $48 \mathrm{~h}$ at two different oxygen levels, $0.5 \%$ and $0.2 \% \mathrm{O}_{2}$. Cell viability after hypoxia treatment was compared to normoxic $\left(21 \% \mathrm{O}_{2}\right)$ controls using crystal violet staining. While incubation at $0.5 \% \mathrm{O}_{2}$ showed no difference in cell viability between normoxic and hypoxic cells (Fig. 1A), lower oxygen levels of $0.2 \%$ significantly reduced the viability of both cell types (Supplemental Fig. 1). Further, VEGFA mRNA levels were quantified to control for the induction of hypoxia response genes after $48 \mathrm{~h}$ at $0.5 \% \mathrm{O}_{2}$. VEGFA is a widely expressed, primary target of HIF transcription factors and thus, a commonly used marker for the hypoxia response of cells (Forsythe et al. 1996; Blancher et al. 2000). RT-qPCR confirmed a 3.6-fold or 7.2-fold increase in VEGFA mRNA levels in A549 and MCF-7 cells, respectively (Fig. 1B). Thus, incubation at $0.5 \% \mathrm{O}_{2}$ for $48 \mathrm{~h}$ robustly induces a hypoxia response in both cell types without causing cell death and was therefore used for all further experiments.

For the transcriptome-wide comparison of hypoxia responses, total RNA was extracted from normoxic and hypoxic A549 and MCF-7 cells, depleted from ribosomal RNA and subjected to deep sequencing $(n=2)$. Approximately 100 million reads were obtained for each sample. We detected expression of 18,214 genes in A549 and 18,880 genes in MCF-7 cells, with 15,684 genes shared between both cell types (transcripts per million [TPM] $>1$ in at least one of the samples for each cell type). From these, differential expression analysis identified 2490 genes (16\%) in A549 and 4503 genes (29\%) in MCF-7, which changed significantly in their abundance $(>1.5$-fold, adjusted $P<0.05$, Supplemental Table 1). Gene ontology (GO) analyses of the differentially expressed genes confirmed the expected induction of hypoxia-associated processes like metabolic reprogramming and extracellular matrix remodeling. 

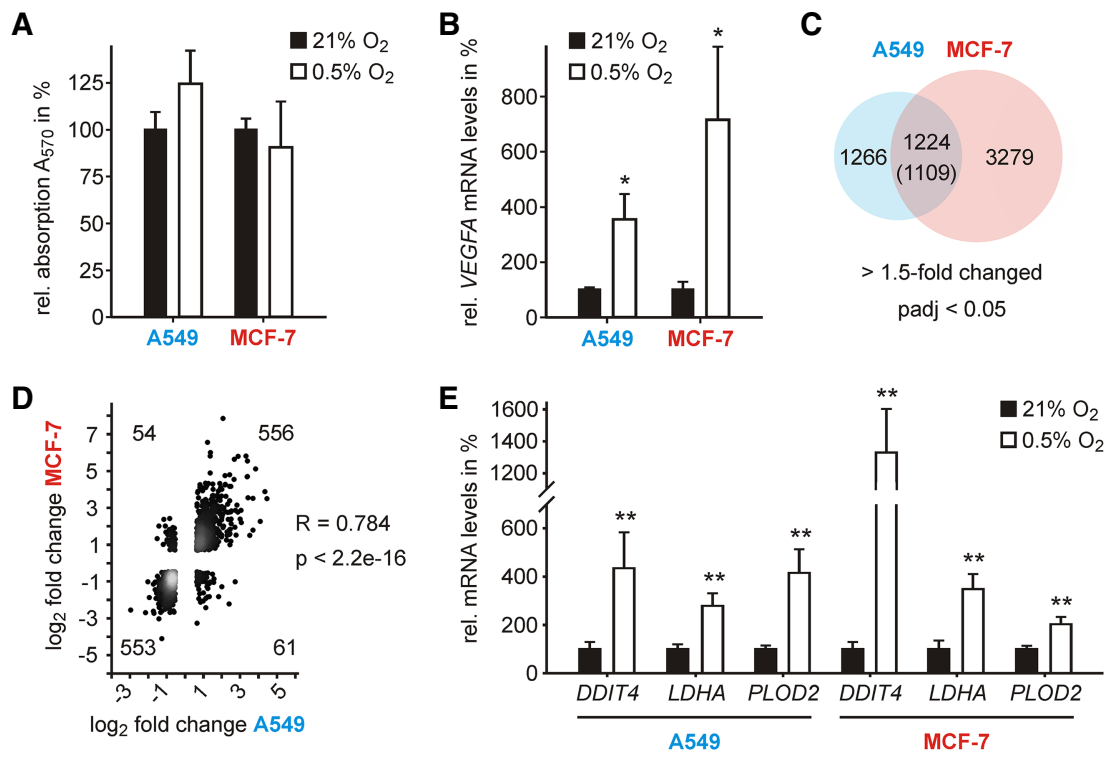

FIGURE 1. Chronic hypoxia induces highly correlated changes in transcript abundance in breast and lung cancer cells. (A) Comparison of the cell viability of A549 and MCF-7 cells cultivated for $48 \mathrm{~h}$ under normoxic $\left(21 \% \mathrm{O}_{2}\right)$ or hypoxic $\left(0.5 \% \mathrm{O}_{2}\right)$ conditions. $n=3$. (B) RT-qPCR quantification of VEGFA mRNA levels in normoxic and hypoxic A549 and MCF-7 cells. Values are normalized to the housekeeping gene RPLPO. $n=3$ (A549) and $n=4$ (MCF-7). (C) Venn diagram showing the overlap of differentially expressed genes in A549 and MCF-7 cells in response to hypoxia. $P<9.52 \times 10^{-124}$ (Fisher's exact test, one-tailed). The number in brackets refers to genes regulated in the same direction. $(D)$ Scatter plot comparing the $\log _{2}$-transformed fold changes (hypoxia/normoxia) of 1224 shared differentially expressed genes in A549 and MCF-7 cells. Pearson correlation coefficient and associated P-value are given. (E) RT-qPCR quantification of the mRNA levels of hypoxia-induced genes in normoxic and hypoxic A549 and MCF-7 cells. Values are normalized to the housekeeping gene RPLPO. $n=5 .\left(^{* *}\right) P<0.01,(*) P<0.05$.

Down-regulated genes were enriched for functions in DNA replication and ribosome biogenesis, reflecting lower energy consumption in response to hypoxia (Supplemental Fig. 2).

Comparison of the differentially expressed genes in the two cell types showed an overlap of 1224 altered genes, which corresponds to almost half of all regulated genes in A549 (49\%) and more than a quarter (27\%) in MCF-7 cells (Fig. 1C). These 1224 genes showed a highly correlated response to hypoxia, with $>90 \%$ regulated in the same direction in both cell types (Fig. 1D). As expected, VEGFA mRNA levels were elevated in both cell types, with a 4.5fold or 6.5-fold increase in A549 and MCF-7 cells, respectively. For further verification, we quantified the induction of three other known hypoxia response genes (DDIT4, LDHA, PLOD2) and confirmed their expected increase in both cell types (Fig. 1E).

Accompanying the changes in transcript abundance, we detected global changes in AS events (absolute delta percent spliced-in $[|\Delta \mathrm{PSI}|] \geq 10 \%$, false discovery rate $[F D R]<0.05$, Supplemental Table 2). Among the detected splicing events, cassette exons (CE) were preferentially altered in response to chronic hypoxia in both, A549 and
MCF-7 cells (Fig. 2A). However, the preferred directionality of the AS events was slightly different between the two cell types. While CE were predominantly skipped in response to hypoxia in MCF-7 cells, A549 cells showed a slight preference for more exon inclusion. In contrast, usage of alternative $5^{\prime}$ and $3^{\prime}$ splice sites as well as complete intron retention was increased in hypoxic MCF-7 cells, but decreased in hypoxic A549 cells (Fig. 2B). Moreover, although the overall number of differentially spliced genes was similar to the number of differentially expressed genes, only 199 shared AS events were detected (Fig. 2C). This corresponds to just $9 \%$ or $5 \%$ of the AS events detected in A549 and MCF-7 cells, respectively. Moreover, of these 199 AS events only 123 (62\%) were regulated in the same direction (Fig. 2D). For verification, we chose eight and seven CE from A549 and MCF-7, respectively. Cassette exons were selected from genes important for tumorigenesis. Comparison of exon inclusion levels with total mRNA levels under normoxia and hypoxia confirmed the expected changes for 10 of the 15 selected events (Supplemental Fig. 3; Supplemental Tables 3, 4). As an example, the reduced inclusion in response to hypoxia of CENPE exon 17 is shown for both cell types in Figure 2E.

In summary, while chronic hypoxia triggers a highly correlated change in transcript abundance within the two cancer types tested, the observed AS changes are substantially different. In addition to the fact that only a small fraction of AS events are common to both cell types, AS events are also frequently changed in the opposite direction.

\section{MBNL2 is induced by chronic hypoxia and facilitates cancer cell adaptation}

Several RBPs and in particular major splicing factors, such as $S R$ proteins and hnRNPs, are associated with cancer progression and metastasis (Anczuków and Krainer 2016). Thus, we were interested in changes of the RBPome in hypoxic A549 and MCF-7 cells. At the transcript level, RBPs are preferentially reduced, most noticeable for core spliceosomal proteins and hnRNPs (Supplemental Table 5).

SR proteins are known to change their phosphorylation status under hypoxia due to the differential expression of 
A

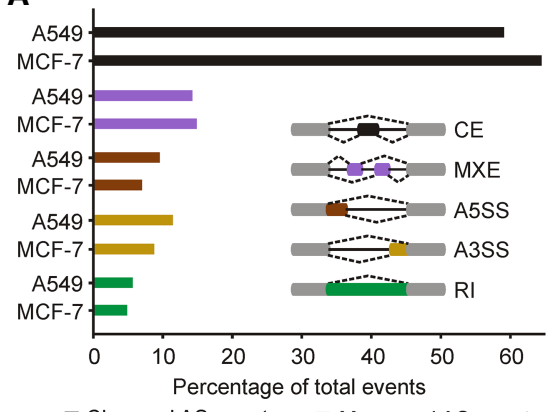

B

E Changed AS events Measured AS events

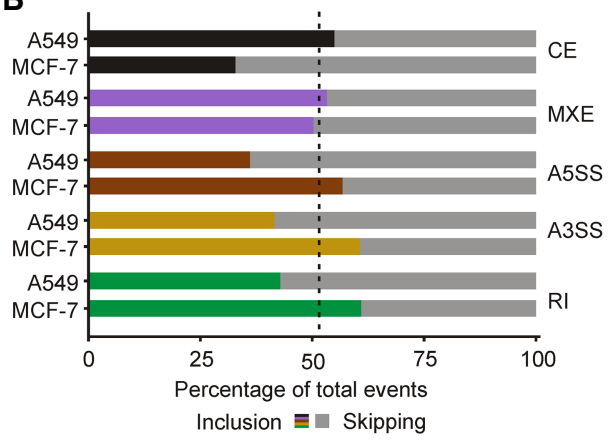

FIGURE 2. Hypoxia-dependent alternative splicing programs are highly cell type-specific. (A) Percentage of significantly altered splicing events $(|\Delta \mathrm{PS}| \mid \geq 10 \%, \mathrm{FDR}<0.05)$ in A549 and MCF-7 cells. Different event types are color coded. (CE) Cassette exon, (MXE) mutually exclusive exons, (A5SS) alternative 5' splice site, (A3SS) alternative $3^{\prime}$ splice site, (RI) retained intron. (B) Percentage of inclusion and skipping for different event types in A549 and MCF-7 cells. (C) Venn diagram showing the overlap of alternative splicing events in A549 and MCF-7 cells in response to hypoxia. Not significant (Fisher's exact test, one-tailed). The number in brackets refers to AS events regulated in the same direction. (D) Scatter plot comparing the $\triangle \mathrm{PSI}$ of shared alternative splicing events in A549 and MCF-7 cells. Pearson correlation coefficient and associated $P$-value are given. (E) RT-PCR of CENPE exon 17 after hypoxia treatment in A549 and MCF-7 cells. The short (118 bp) and long (192 bp) PCR products correspond to exon 17 exclusion and inclusion, respectively. $n=2$. (N) Normoxia, $(H)$ hypoxia, $(M)$ size marker.

stability. In addition to splicing factors, like SR proteins and hnRNPs, changes in core spliceosomal proteins will affect splicing changes. Thus, we examined the mRNA levels of four core spliceosomal proteins (LSM4, SF3B4, SNRPC, and SNRPD1) and, similar to hnRNPs, found a more pronounced reduction of core spliceosomal factor mRNA levels in MCF-7 cells (Supplemental Fig. 5). This finding is in line with the increased exon skipping and intron retention specifically detected in hypoxic MCF-7 cells, suggesting an overall lower splicing efficiency of hypoxic MCF-7 compared to hypoxic A549 cells.

Among the few elevated RBPs under hypoxia, MBNL2 caught our interest as it has recently been shown to be elevated in ccRCC (clear cell renal cell carcinoma) and HCC (hepatocellular carcinoma) patient samples (Lee et al. 2016; Perron et al. 2018). We verified increased mRNA and protein levels of MBNL2 in A549 and MCF-7 cells (Fig. 4A,B; Supplemental Fig. 6). Interestingly, its paralogs MBNL1 and MBNL3 were unaffected or even decreased at mRNA and protein level (Fig. 4A,B; Supplemental Fig. 7), suggesting a specific role of MBNL2 in hypoxia adaptation. Since MBNL2 was described as an oncogene in

SR protein kinases (Jakubauskiene et al. 2015; Bowler et al. 2018). Thus, we focused on the hypoxia response of hnRNPs, which has not been analyzed so far. We could show reduced mRNA levels of all six tested hnRNPs, hnRNP A1, C, DL, E2, L, and M, under hypoxia in MCF-7 cells (Fig. 3A). In A549 cells, hnRNPA1 and M mRNA levels were significantly reduced under hypoxia, while the other tested hnRNPs showed only a trend toward mRNA level reduction. Since changes in mRNA levels do not necessarily reflect altered protein levels and vice versa, we also examined the protein levels of hnRNPs under hypoxia. Western blot analyses confirmed reduced protein levels of all six hnRNPs in both cell types (Fig. 3B). Mechanistically, these may result from overall reduced transcript levels or a shift of AS toward NMD-sensitive isoforms as shown for hnRNP DL in MCF-7 cells (Supplemental Fig. 4; Kemmerer et al. 2018). For some hnRNPs in A549 cells, however, our analysis did not reveal any changes in transcript abundance or AS events, indicating further regulatory mechanisms, such as lower translation efficiency or protein
ccRCC (Perron et al. 2018), but as a tumor suppressor in HCC (Lee et al. 2016), we investigated the influence of MBNL2 expression on the cell viability of hypoxic A549 and MCF-7 cells. We performed an siRNA-mediated, transient knockdown of MBNL2 in both cell types and quantified cell viability in the presence and absence of the chemotherapeutic drug cisplatin using crystal violet staining. Cisplatin concentrations were chosen to induce only low levels of cell death under normoxic conditions, allowing for the detection of changes in cisplatin sensitivity upon hypoxia and/or knockdown of MBNL2 (Supplemental Fig. 8A). MBNL2 knockdown induced cell death in hypoxic A549, both with or without cisplatin-treatment (Fig. 4C; Supplemental Table 6). Moreover, knockdown of MBNL2 had a stronger effect on A549 cell viability under hypoxia compared to normoxia in all tested conditions (Supplemental Fig. 8B). In addition, migration of hypoxic A549 cells was reduced after knockdown of MBNL2 (Fig. 4D,E). In hypoxic MCF-7 cells, MBNL2 knockdown increased cisplatin-induced cell death at $20 \mu \mathrm{M}$ (Fig. 4C). 


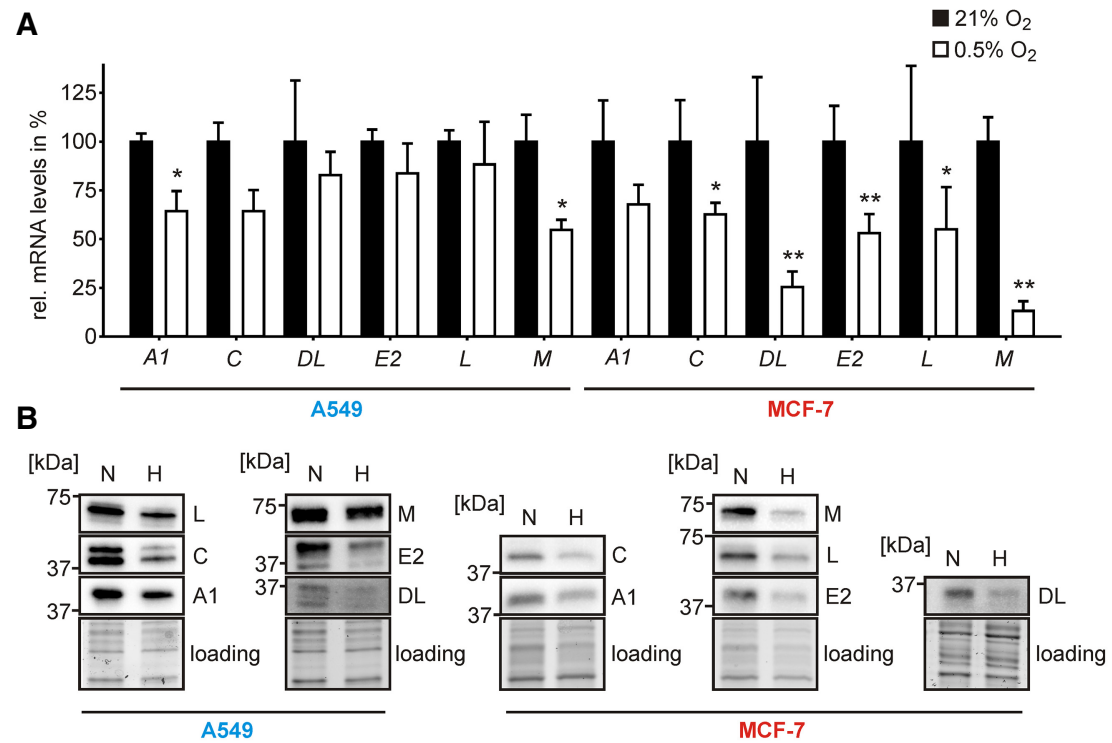

FIGURE 3. Chronic hypoxia reduces hnRNP mRNA and protein levels. (A) RT-qPCR quantification of hnRNP A1, C, DL, E2, L, and M mRNA levels in normoxic and hypoxic A549 and MCF-7 cells. Values are normalized to the housekeeping gene RPLPO. $n=3-5$. (B) Western blot of hnRNPs A1, C, DL, E2, L, and M in normoxic and hypoxic A549 and MCF-7 cells. Anti-hnRNP antibodies were used to visualize the respective protein levels. $n=4$. els. These shared genes were preferentially changed in the opposite direction (71\%) (Fig. 5C), supporting a role of MBNL2 induction in the regulation of hypoxia response genes.

Interestingly, a similar proportion of shared genes was found when comparing only to genes that were regulated consistently in hypoxic A549 and MCF-7 cells (351 out of 1,109 genes, 32\%; Supplemental Fig. 11A; Supplemental Table 7), suggesting a cell type-independent function of MBNL2 in controlling hypoxia adaptation. Noticeably, these shared differentially expressed genes were mostly increased in response to hypoxia and decreased after knockdown of MBNL2, including several HIF target genes (Supplemental Fig. 11B).

This suggests that one function of MBNL2 induction might be to further increase the mRNA levels of HIF target genes. To test this hypothesis, we verified by RT-qPCR that the Further, knockdown of MBNL2 reduced cell viability of hypoxic, but not normoxic MCF-7 cells in the absence of cisplatin (Supplemental Fig. 8B). Together, these results suggest that MBNL2 induction supports the adaptation of breast and lung cancer cells to hypoxia.

\section{MBNL2 controls the transcript abundance of hypoxia response genes}

To gain insight into the molecular mechanism by which MBNL2 facilitates the adaptation of cancer cells to hypoxia, we performed a transcriptomic analysis after the knockdown of MBNL2 in hypoxic MCF-7 cells (Fig. 5A; Supplemental Fig. 9). Total RNA was extracted from hypoxic MCF-7 cells transfected with an siRNA targeting MBNL2 (siMBNL2) or a nonsilencing control siRNA (siCTRL), poly(A)-selected and subjected to deep sequencing $(n=2)$. Approximately 80 million reads were obtained for each sample. A total of $4370(27 \%)$ genes were differentially expressed after knockdown of MBNL2 (>1.5-fold, adjusted $P<0.05$, Supplemental Table 7). GO analysis of these genes showed an enrichment in hypoxia response genes, including genes related to glucose metabolism, emphasizing a contribution of MBNL2 to hypoxia adaptation (Supplemental Fig. 10).

Comparison of the differentially expressed genes after MBNL2 knockdown with the hypoxia response of MCF-7 cells showed 1528 shared regulated genes (Fig. 5B; Supplemental Table 7). Thus, about a third of all hypoxiaregulated genes in MCF-7 cells are affected by MBNL2 lev-
mRNA levels of HIF targets were reduced after the knockdown of MBNL2. Indeed, knockdown of MBNL2 significantly attenuated the hypoxia-dependent induction of the HIF target genes ALDOC, ENO2, ITGA5, LOX, and VEGFA in MCF-7 cells (Fig. 5D). Likewise, a reduced induction of ALDOC, ITGA5, and VEGFA after MBNL2 knockdown was detected in A549 cells (Fig. 5D).

In order to investigate whether our findings from the cell lines are relevant in cancer patients, we used mRNA level data from patients with breast invasive carcinoma (BRCA; 1088 patients) and lung adenocarcinoma (LUAD; 533 patients) of The Cancer Genome Atlas (TCGA) PanCancer Atlas (Hoadley et al. 2018). We detected significant positive correlations of MBNL2 mRNA levels with the mRNA levels of the hypoxia response genes VEGFA, ITGA5, and LOX in BRCA patients and LOX in LUAD patients (Supplemental Fig. 12). Further, MBNL2 mRNA levels were positively correlated with HIF1A and HIF2A mRNA levels, suggesting that MBNL2 induction might also depend on hypoxia signaling in cancer patients (Supplemental Fig. 12). More globally, when comparing hypoxia response genes, that is, genes that were significantly induced in A549 and MCF-7 in response to hypoxia (Fig. 1D), to all other genes in the TCGA data sets, we found a significant trend toward positive correlation with MBNL2 mRNA levels (Supplemental Fig. 13; Supplemental Table 8). Together, these observations support that MBNL2 controls the transcript abundance of several HIF target genes under hypoxia, with potential implications in human cancer patients. 
A
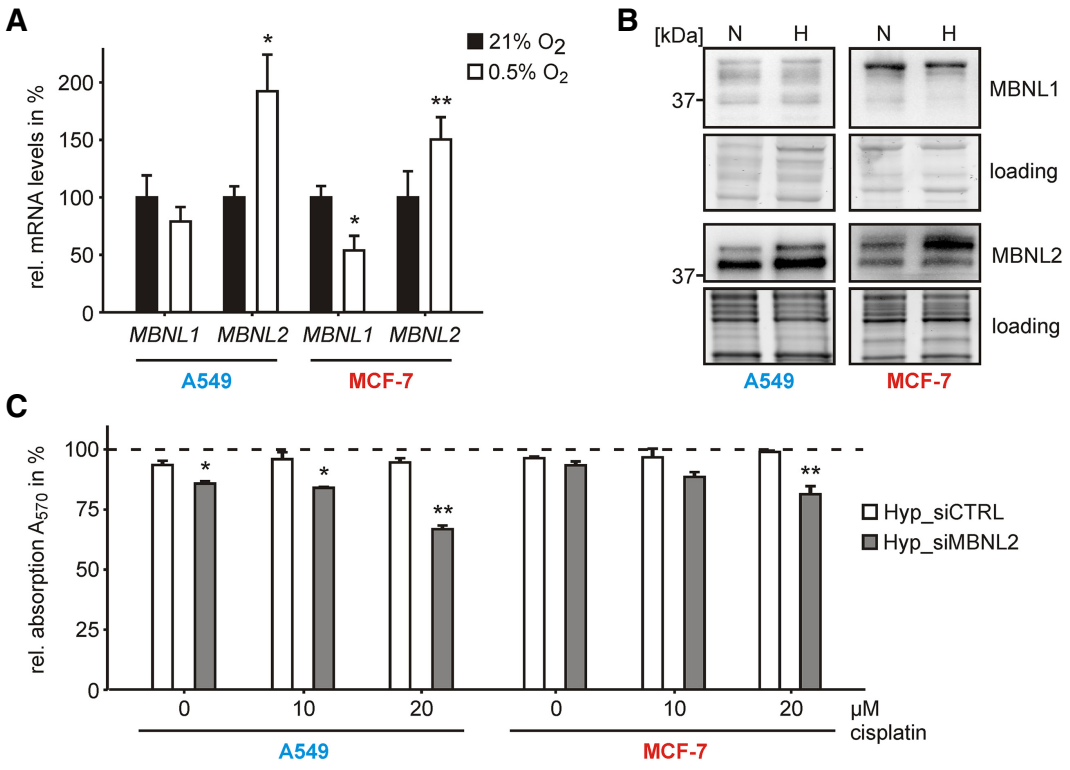

D

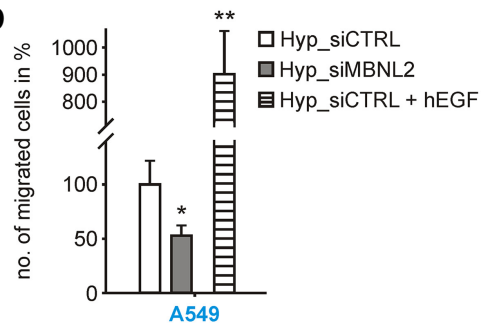

E

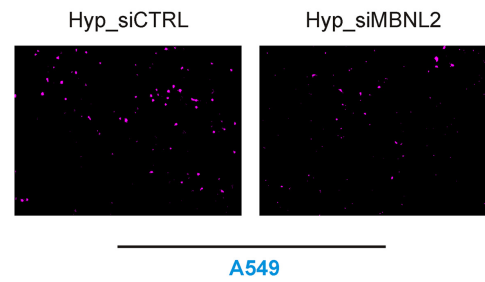

FIGURE 4. MBNL2 is specifically induced by chronic hypoxia and contributes to the adaptation of cancer cells. (A) RT-qPCR quantification of the mRNA levels of MBNL1 and MBNL2 in normoxic and hypoxic A549 and MCF-7 cells. Values are normalized to the housekeeping gene RPLPO. $n=4$. (B) Western blot of MBNL1 and MBNL2 in normoxic and hypoxic A549 and MCF-7 cells. Anti-MBNL1 and anti-MBNL2 antibodies were used to visualize the respective protein levels. Total lane protein is shown as loading control. $n=2$. (N) Normoxia, $(\mathrm{H})$ Hypoxia. (C) Cell viability of hypoxic A549 and MCF-7 cells after knockdown of MBNL2. Cells were additionally treated with the indicated concentrations of cisplatin for $24 \mathrm{~h}$. Absorption was normalized to normoxic control cells with the respective cisplatin concentration. $n=3$. (D) Migration assay of hypoxic A549 cells after knockdown of MBNL2. As positive control, cells were stimulated with $100 \mathrm{ng} / \mu \mathrm{L}$ hEGF. $n=3$. (E) Representative pictures used to quantify the numbers of migrated A549 cells in $D .\left(^{* *}\right) P<0.01,\left(^{*}\right) P<0.05$.

In order to test whether the paralog MBNL1 is also able to control the transcript abundance of HIF target genes, we performed an siRNA-mediated knockdown of MBNL1 under hypoxia (Fig. 5E). Interestingly, knockdown of MBNL1 did not lead to a reduction of HIF target mRNA levels, but to an increase (Fig. 5F). This increase is most likely the result of an associated increase in MBNL2 levels (Fig. 5E), due to cross-regulation between the two paralogs (Konieczny et al. 2018). This underlines the specific role of MBNL2 in the hypoxia response of cancer cells.

\section{MBNL2 binds to the VEGFA $3^{\prime}$ UTR and controls the level of secreted VEGF-A protein}

It has been suggested that MBNL2 stabilizes mRNAs from correlation of MBNL2 mRNA levels with putative target
mRNA levels across a panel of different cell types and tissues (Ray et al. 2013). However, knockdown studies showed no global decrease in target mRNA levels, but suggested involvement of MBNL2 in mRNA localization, in particular of secreted proteins, thereby promoting efficient protein secretion (Wang et al. 2012). Thus, we tested whether MBNL2 affects the stability of the VEGFA mRNA and/or the secretion of VEGFA protein.

A direct interaction of MBNL2 with the VEGFA mRNA was confirmed by MBNL2 immunoprecipitation experiments (Perron et al. 2018). Since CLIP-seq data mostly showed binding of MBNL2 to 3'UTRs (Charizanis et al. 2012), we first tested whether MBNL2 is able to directly recognize the $3^{\prime} U T R$ of VEGFA. Thus, we examined the 3'UTR of VEGFA for possible MBNL2 binding sites, that is, two clustered 5'-YGCY-3' motifs (Lambert et al. 2014). We identified three potential binding sites in the $3^{\prime} U T R$ of the VEGFA mRNA (Supplemental Fig. 14). These potential binding sites, plus 40-60 nt flanking sequences, were cloned in place of the natural MBNL binding site of the Atp2a1 minigene, an established test system for MBNL binding, based on the differential inclusion of Atp2a1 alternative exon 22 (Cywoniuk et al. 2017). In this system, exon 22 inclusion levels are a measure of MBNL binding activity (Supplemental Fig. 15). Alternative exon 22 splicing is analyzed after plasmid-based expression of MBNLs in a MEF (mouse embryonic fibroblast) cell line with a genetic knockout of both, Mbn/1 and Mbn/2. This allows for individual testing of the binding behavior of different MBNL paralogs and their various isoforms.

The expression pattern of the different MBNL isoforms depends on tissue type and developmental stage (Wang et al. 2012). The different isoforms show distinct characteristics with respect to their cellular localization, RNA binding affinity and splicing regulation (Sznajder et al. 2016). MBNL2 in particular contains two alternatively spliced exons in its coding sequence giving rise to four protein isoforms ranging from 38 to $41 \mathrm{kDa}$ (Sznajder et al. 2016). MBNL2 isoform 40 (MBNL2-40) was chosen for binding site assessment in this study, because it is the most 
A

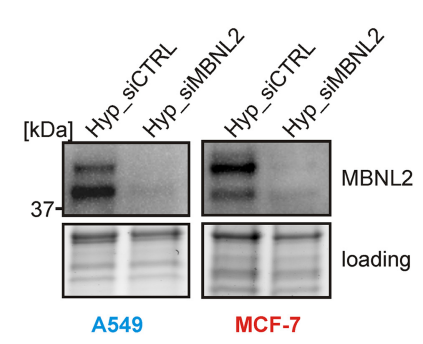

B Differentially expressed genes in MCF-7

SiMBNL2 Hypoxia

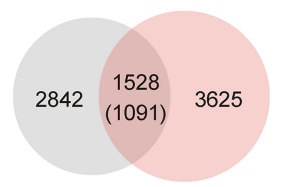

$>1.5$-fold changed padj $<0.05$
C

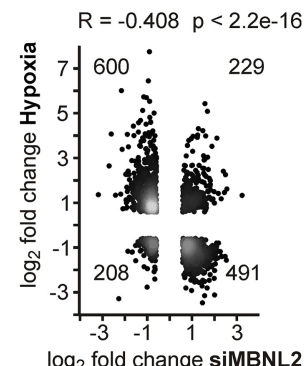

$\frac{*}{\prod_{5}}$

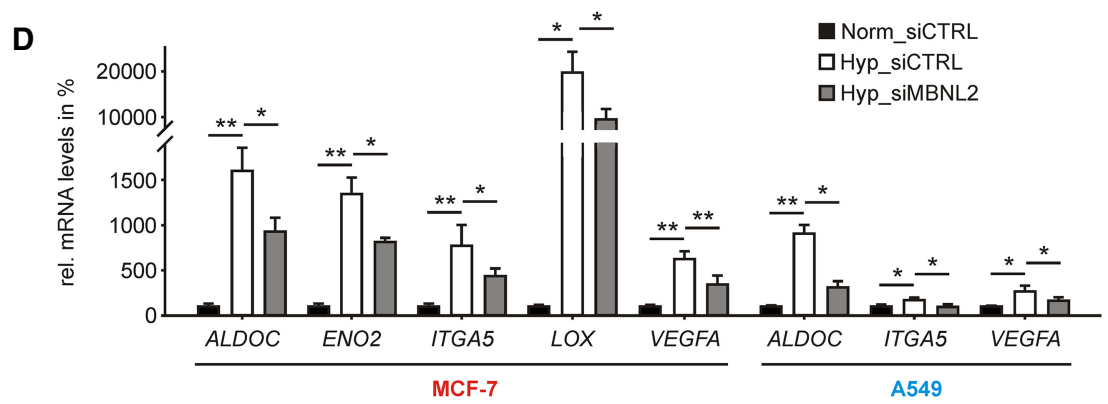

E

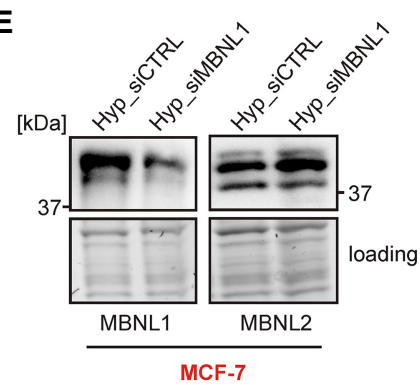

$\mathbf{F}$

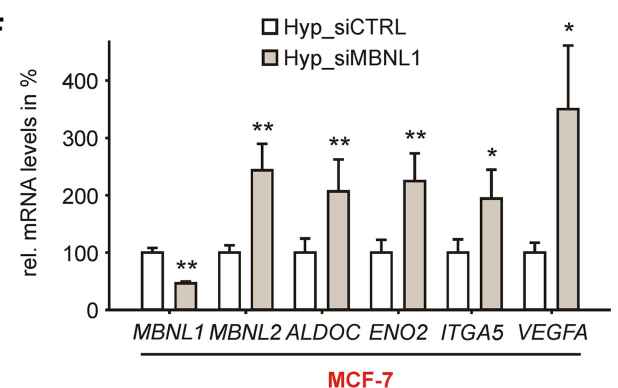

FIGURE 5. MBNL2 controls the hypoxia response of breast and lung cancer cells by regulating transcript abundance. (A) Western blot of MBNL2 after siRNA-mediated knockdown in hypoxic A549 and MCF-7 cells. Anti-MBNL2 was used to verify the respective knockdown. Total lane protein is shown as loading control. $n=3-4$. (B) Venn diagram showing the overlap of differentially expressed genes after knockdown of MBNL2 and in response to hypoxia in MCF-7. $P<$ $1.61 \times 10^{-37}$ (Fisher's exact test, one-tailed). The number in brackets refers to genes regulated in the opposite direction. (C) Scatter plot comparing the fold changes of shared differentially expressed genes after knockdown of MBNL2 and in response to hypoxia in MCF-7. (D) RTqPCR quantification of the mRNA levels of HIF target genes in normoxic and hypoxic A549 and MCF-7 cells and after knockdown of MBNL2. Values are normalized to the housekeeping gene RPLPO. $n=3-6$. (E) Western blot of MBNL1 and MBNL2 after siRNA-mediated knockdown of MBNL1 in hypoxic MCF-7 cells. Anti-MBNL1 and anti-MBNL2 were used to verify the respective protein levels. Total lane protein is shown as loading control. $n=3$. (F) RTqPCR quantification of the mRNA levels of MBNL1, MBNL2, and HIF target genes. $n=3$. $\left({ }^{* *}\right)$ $P<0.01$. (*) $P<0.05$.

efficient splicing regulator of the four MBNL2 protein isoforms (Sznajder et al. 2016) and the most abundant isoform detected in MCF-7 cells (Fig. 4B). MBNL1 isoform 42 was chosen for comparison because MBNL1-42 is the corresponding isoform to MBNL2-40, containing a similar sequence composition encoded by the same alternative exons (Sznajder et al. 2016) and is also abundantly expressed in MCF-7 cells (Fig. 4B).

The naturally encoded MBNL binding site present within the Atp2a1 minigene served as a positive control and led to an increased inclusion of exon 22 after expression of MBNL2-40, while inclusion was reduced when this binding site was deleted from the minigene (Supplemental Fig. 16). The inclusion of exon 22 was restored after MBNL2-40 expression and insertion of VEGFA binding sites 1 or 3 into the minigene, less so with binding site 2 (Supplemental Fig. 16). Equal overexpression efficiencies (Supplemental Fig. 17) allowed us to compare the splicing activity and thus, the VEGFA 3'UTR binding capacity, of MBNL240 and MBNL1-42. Comparing the $\triangle \mathrm{PSI}$ values for the positive control, which contains the high affinity Atp2a1 binding site, showed a higher splicing regulatory activity of MBNL142, compared to MBNL2-40 (Fig. 6A). This observation is consistent with previous experiments showing that MBNL1 isoforms are more active splicing regulators than the corresponding MBNL2 isoforms (Sznajder et al. 2016). Strikingly, using the VEGFA binding site 1, MBNL2-40 was similarly efficient in restoring exon 22 inclusion as in the positive control, suggesting that MBNL2-40 could bind to the VEGFA $3^{\prime} U T R$ with high affinity. In contrast, MBNL1-42 was not able to restore exon 22 inclusion with any of the three VEGFA binding sites to the level of the positive control, indicating that it might bind to the VEGFA $3^{\prime}$ UTR with lower affinity (Fig. 6A).

In order to examine the binding affinities of MBNL2 and MBNL1 to the VEGFA 3'UTR directly, we quantified the interaction between the two MBNL paralogs and the three RNA binding sites from the VEGFA $3^{\prime}$ UTR in vitro. MBNL2 showed an 11-fold higher binding affinity for VEGFA binding site 1 than MBNL1 (Fig. 6B; Supplemental Fig. 18), underlining the results from the Atp2a1 splicing assay (Fig. 6A). Thus, MBNL2 is able to directly recognize the $3^{\prime} U T R$ of VEGFA and has a higher binding affinity to binding site 1 than its paralog MBNL1.

Next, we tested whether MBNL2 stabilizes the MRNA of VEGFA. Thus, we measured changes in mRNA half-life after knockdown of MBNL2 in hypoxic MCF-7 cells. Actinomycin $D$ assays showed no change in VEGFA mRNA half-life in response to MBNL2 knockdown 
A

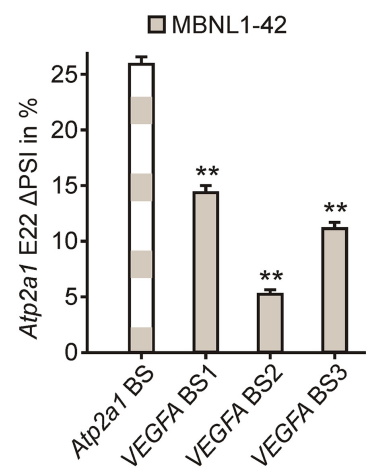

B

$$
\begin{aligned}
& \diamond(\text { CUG })_{20} \\
& -\checkmark \text { VEGFA BS1 } \\
& -\square \text { VEGFA BS2 } \\
& -\square \text { VEGFA BS3 }
\end{aligned}
$$
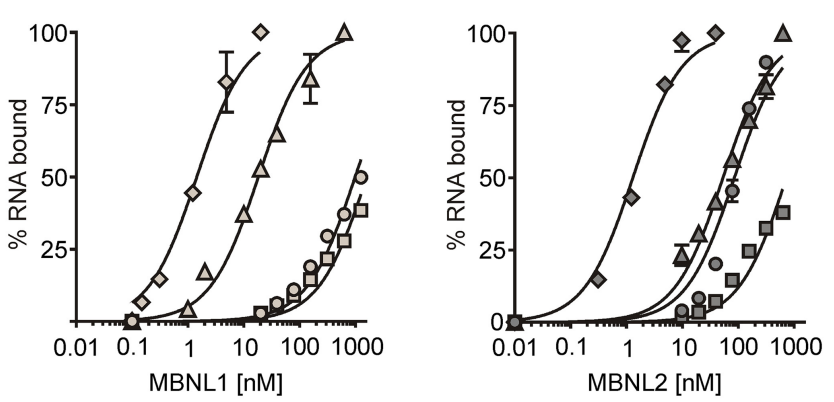

口MBNL2-40

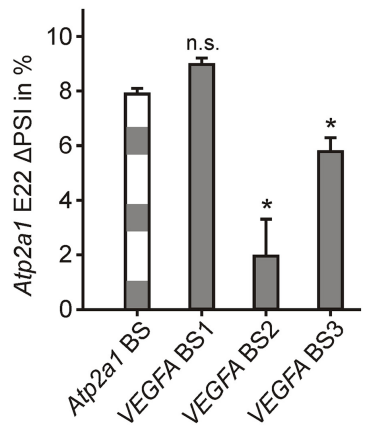

$\diamond(\mathrm{CUG})_{20}$

- - VEGFA BS1

마 VEGFA BS2

$\triangle$ VEGFA BS3
C

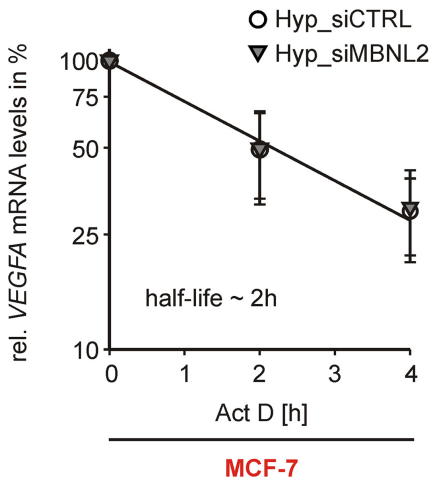

\begin{tabular}{c|c|c|c|c}
$\mathrm{K}_{\mathrm{D}}$ & (CUG $_{20}$ & VEGFA BS1 & VEGFA BS2 & VEGFA BS3 \\
\hline MBNL1 & $1.4 \pm 0.1$ & $982.8 \pm 55.5$ & $1592 \pm 101$ & $18.2 \pm 1.1$ \\
\hline MBNL2 & $1.3 \pm 0.1$ & $84.7 \pm 9.3$ & $725 \pm 50$ & $53.0 \pm 3.3$
\end{tabular}

D

norm_siCTRL 口Hyp_siCTRL

口Hyp_siMBNL2

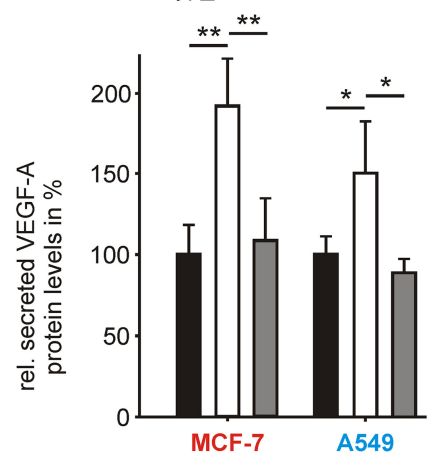

FIGURE 6. MBNL2 binds to the $3^{\prime} U T R$ of VEGFA and controls secreted VEGF-A protein levels. (A) RT-PCR quantification showing different Atp2a1 exon 22 (E22) inclusion levels depending on inserted binding sites (BS) from the VEGFA $3^{\prime} U T R$ and MBNL2 isoform 40 (MBNL2-40) or MBNL1 isoform 42 (MBNL1-42) expression in Mbnl1 and 2 double knockout MEF cells. Shown are $\triangle \mathrm{PSI}$ values with respect to the negative control in which the binding site is deleted (see also Supplemental Fig. 15). Statistical significance is given in reference to the inclusion level observed with the natural Atp2a1 binding site. $n=3$. (B) Relative amounts of MBNL1 and MBNL2 bound to the VEGFA $3^{\prime} U T R$ RNA and calculated $K_{D}$ values. RNA carrying $(C U G)_{20}$ repeats was used as positive control. $n=3$. (C) Half-life of VEGFA mRNA. MCF-7 cells were treated with $5 \mu \mathrm{g} / \mathrm{mL}$ actinomycin D (ActD). Thereafter, total RNA was isolated at $2 \mathrm{~h}$ intervals and VEGFA mRNA levels were quantified by RT-qPCR. VEGFA mRNA level was normalized to the housekeeping gene RPLPO. $n=3$. (D) Quantification of secreted VEGF-A protein levels by ELISA. Secreted VEGF-A levels were determined in the supernatants of normoxic and hypoxic A549 and MCF-7 cells and after knockdown of MBNL2. $n=4$. $\left(^{* *}\right) P<0.01 .\left(^{*}\right) P<0.05$.

(Fig. 6C). We then tested if MBNL2 affects VEGF-A protein levels and quantified secreted VEGF-A by ELISA (Fig. 6D). Knockdown of MBNL2 completely suppressed hypoxia-dependent induction of secreted VEGF-A protein in both, A549 and MCF-7 cells. Thus, regulation at protein level is more pronounced than regulation at mRNA level. In sum, MBNL2 directly recognizes the 3'UTR of VEGFA. Our results indicate that it does not affect the stability of VEGFA mRNA, but possibly affects secretion of the VEGF-A protein.

\section{MBNL2 controls hypoxia- dependent AS}

Along with the changes in transcript abundance, we also detected 2074 MBNL2-dependent changes in AS events $(|\Delta P S| \mid \geq 10 \%$, FDR $<0.05$, Supplemental Table 9). For verification, we chose six CE sensitive to MBNL2 knockdown. Cassette exons were selected from genes important for tumorigenesis. Comparison of exon inclusion levels with total mRNA levels with and without knockdown of MBNL2 in hypoxic MCF-7 cells, confirmed the expected changes for five of the six selected events (Supplemental Fig. 19; Supplemental Table 10).

Comparison with the splicing changes during hypoxia showed 393 shared events, corresponding to $19 \%$ or $9 \%$ of the events detected after knockdown of MBNL2 or hypoxia treatment of MCF-7 cells, respectively (Fig. 7A). As for the differential expression described above, these 393 AS events were preferentially regulated in the opposite direction (89\%), suggesting that MBNL2 induction contributes to hypoxia-dependent alternative splicing (Fig. 7B). The majority of these were exon skipping events in which the inclusion of CE is reduced in response to hypoxia. These are increased again after MBNL2 knockdown, indicating a repressive role of MBNL2. This is exemplified by PIGN exon 12, which inclusion is reduced 


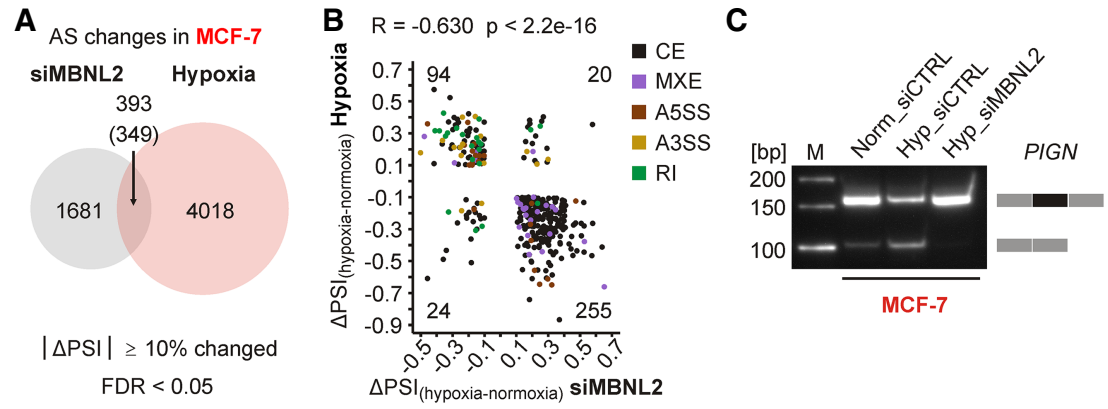

FIGURE 7. MBNL2 controls the hypoxia-dependent alternative splicing of CE. (A) Venn diagram showing the overlap of AS changes after knockdown of MBNL2 and in response to hypoxia in MCF-7. Not significant (Fisher's exact test, one-tailed). The number in brackets refers to AS events regulated in the opposite direction. (B) Scatter plot comparing the fold changes of shared AS events after knockdown of MBNL2 and in response to hypoxia in MCF-7. Pearson correlation coefficient and associated P-value are given. (C) RT-PCR of PIGN exon 12 in normoxic and hypoxic MCF-7 cells and after knockdown of MBNL2. The short isoform (100 bp) corresponds to exon 2 exclusion, the long isoform (160 bp) to exon 12 inclusion. $n=2$. (M) Size marker.

in response to hypoxia. Inclusion was sustained after simultaneous knockdown of MBNL2 (Fig. 7C), demonstrating that modulation of MBNL2 levels is sufficient to reverse hypoxia-induced splicing changes in MCF-7 cells. PIGN catalyzes one step in the synthesis of GPI (glycosylphosphatidylinositol)-anchors of membrane proteins. The alternatively spliced exon 12 is located in the catalytic domain and may thus affect the enzymatic function of PIGN. Interestingly, a reduction of PIGN function through alternative splicing has been shown to cause genetic instability and thus promote leukemic progression in acute myeloid leukemia (Teye et al. 2017). Moreover, PIGN levels are altered by alternative splicing of another CE in endothelial cells in response to hypoxia (Weigand et al. 2012), pointing to a more widespread function of PIGN in hypoxia adaptation.

In sum, MBNL2 promotes the response of cancer cells to hypoxia by controlling the mRNA levels of HIF target genes. Thus, the expression of genes that determine hypoxia-driven angiogenic, metastatic and metabolic reprogramming of cancer cells is collectively regulated by MBNL2. In addition, MBNL2 contributes to hypoxiadependent $A S$, in particular by reducing the inclusion of CE.

\section{DISCUSSION}

Hypoxia is an important modulator of tumorigenesis and metastasis. It is a common characteristic of a tumor's microenvironment and alters gene expression in affected cells. Cellular adaptation to hypoxia enables to sustain proliferation, escape apoptosis and initiate angiogenesis, ultimately promoting tumorigenesis. So far, proteins that are targeted in cancer therapies mostly belong to the HIF signaling pathway (for review, see Wilson and Hay 2011). However, while earlier studies focused mainly on transcriptional changes induced by HIF transcription factors, post-transcriptional regulation has recently been identified as a critical factor in the adaptation and survival of cancer cells during hypoxia (Oltean and Bates 2014; Gallego-Paez et al. 2017; Khabar 2017; Escobar-Hoyos et al. 2019; Robichaud et al. 2019). Such post-transcriptional regulation is controlled by RBPs, rendering them new potential targets for cancer therapeutics.

Comparing the hypoxic responses of breast and lung cancers, the most common cause of cancer-related deaths in males and females, respectively, we observed a specific increase in MBNL2 levels, while other splicing factors are generally reduced. MBNL proteins have just recently been shown to be potent regulators of tumor progression. MBNL1 contributes to colorectal carcinogenesis via interference with Dicer1 recruitment to miRNA precursors (Tang et al. 2015). MBNL2 is increased in clear cell renal cell carcinoma (ccRCC) and can act as an oncogenic driver (Perron et al. 2018). MBNL3 was found to promote hepatocarcinogenesis (Yuan et al. 2017). In contrast, MBNL1 and MBNL2 were also described to suppress metastasis (Fish et al. 2016; Tang et al. 2019; Zhang et al. 2019). For MBNL1 was proposed that these contrasting roles might depend on the levels of specific isoforms (Tabaglio et al. 2018).

The observed increase of MBNL2 in renal carcinoma is particularly interesting as ccRCC usually involves loss of the Von Hippel-Lindau tumor suppressor (VHL) protein (Meléndez-Rodríguez et al. 2018). Under normoxic conditions, VHL targets hydroxylated HIF $\alpha$ subunits for polyubiquitination and subsequent degradation by the proteasome (Gossage et al. 2015). Thus, the HIF pathway is constitutively activated and might be responsible for induction of MBNL2. In addition, elevated MBNL2 mRNA levels were detected in PyMT mouse breast cancer cells after chronic and intermittent hypoxia (Chen et al. 2018). Together with our study, these data suggest that MBNL2 activation in hypoxia is widespread in cancer and modulates tumorigenesis and metastasis.

Our study shows MBNL2-dependent VEGFA expression in breast and lung cancer cells. In accordance, VEGFA mRNA levels along with four other hypoxia response genes were strongly correlated with MBNL2 mRNA levels in ccRCC tumor samples (Perron et al. 2018). This suggests that MBNL2 universally controls the hypoxia response of various cancer types. Under hypoxia, VEGFA expression is induced by HIF1, resulting in neoangiogenesis, promoting proliferation and cell migration, and ultimately tumor growth (Yancopoulos 2010). Accordingly, it is 
therapeutically targeted using monoclonal antibodies and tyrosine kinase inhibitors in various cancers. In addition to the transcriptional control, VEGF-A levels are tightly controlled also at the post-transcriptional level (for review, see Arcondeguy et al. 2013). The translation of VEGF-A is regulated by two internal ribosome entry sites (IRESs), two alternative CUG initiation codons and a GAIT element (interferon- $\gamma$-activated translation inhibitor) in its $3^{\prime}$ UTR (Yao et al. 2013; Arif et al. 2018). The stability of the VEGFA mRNA is controlled by several AU-rich elements (AREs) and a CA-rich element (CARE) that is bound by miRNAs under normoxia (Jafarifar et al. 2011). Under hypoxic conditions, nuclear hnRNP $L$ is translocated into the cytoplasm, competes with miRNA binding and thus stabilizes the VEGFA mRNA. The three MBNL2 binding sites in the VEGFA 3'UTR proposed in this study do not overlap with the previously identified binding sites of RBPs or miRNAs. Thus, we have identified an additional, independent layer in VEGFA regulation by MBNL2.

Binding assays showed that MBNL2-40 is most likely to bind the VEGFA 3'UTR binding site 1 (Fig. 6A,B). Mechanistically, we could rule out a stabilizing effect of MBNL2 on the VEGFA mRNA in actinomycin D assays. Instead, we found that MBNL2 knockdown suppressed VEGF-A protein secretion. Accordingly, "SRP-dependent cotranslational targeting of proteins to the membrane" was the most significant GO term of reduced genes after MBNL2 knockdown in MCF-7 cells (see Supplemental Fig. 10). In line with our findings, MBNLs have been shown to regulate mRNA localization, particularly of secreted proteins (Du et al. 2010; Wang et al. 2012). An mRNA-stabilizing role of MBNL2 has not yet been experimentally confirmed. However, we cannot exclude the possibility that MBNL2dependent stabilization of specific mRNAs contributes to its role in hypoxia adaptation. Interestingly, MBNL1dependent destabilization of mRNA targets was shown (Masuda et al. 2012). MBNL2 induction in hypoxia could lead to displacement of MBNL1 and thus to MRNA stabilization of target mRNAs other than VEGFA.

Notably, the knockdown of MBNL1 showed an opposite effect to the knockdown of MBNL2 during hypoxia. Since all studies so far have found a broad overlap of the binding specificities of MBNL1 and MBNL2, it is difficult to rationalize how the function of the two proteins can be separated. One possibility might be that slight changes in affinity for certain mRNAs, as suggested by our binding affinity assay, could be sufficient to promote preferential association with one paralog in vivo. Further possibilities could be different cellular localization, different association with target mRNAs, or differential interaction with other proteins during hypoxia. Alternatively, the two paralogs could in principle both stimulate the translation of secreted proteins, but with different efficiencies. Thus, a change in the ratio of the two paralogs can lead to seemingly opposite effects, depending on whether the more active paralog is in- duced or reduced. Such a competitive mode of action has been demonstrated for MBNLs in splicing regulation (Taylor et al. 2018).

In sum, MBNL2 governs the expression of the therapeutic target VEGFA in various cancers, indicating a universal, cell type-independent control. Moreover, since its paralogs are not induced by hypoxia, MBNL2 plays a unique role in hypoxia adaptation, crucial for the survival of cancer cells. Remarkably, TCGA data set analyses revealed a predominant positive correlation of MBNL2 expression with hypoxia response genes in breast and lung cancer samples, suggesting that MBNL2 affects hypoxia adaptation also in cancer patients.

In addition to changes in transcript abundance, we found that MBNL2 also controls hypoxia-dependent changes in alternative splicing. Preferably, the inclusion of CE reduced under hypoxia was restored by the knockdown of MBNL2, suggesting a mostly repressive function of MBNL2 in the splicing response to hypoxia. This is not limited to hypoxia-regulated AS, as increased CE inclusion was the predominant splicing event observed in hypoxic MCF-7 cells after knockdown of MBNL2. In contrast, previous studies found equal numbers of induced and reduced CE after MBNL protein depletion (Du et al. 2010; Charizanis et al. 2012). It is still unclear how exactly MBNL proteins control alternative splicing. Thus, the observed suppressive effect of MBNL2 may be linked to the widespread changes observed within the splicing machinery in response to chronic hypoxia.

Widespread reduction in splicing-associated proteins has been demonstrated as a general stress response of cancer cells after different types of chemotherapy (Anufrieva et al. 2018). In addition, altered SR protein activity was reported in hypoxic prostate cancer (Bowler et al. 2018) and HeLa cells (Jakubauskiene et al. 2015). Together with the widespread reduction of hnRNPs identified here, we suggest global effects of hypoxia on the splicing machinery. Interestingly, this change does not lead to a global retention of introns, as previously observed in response to acute hypoxia (up to 24 h) (Brady et al. 2017; Han et al. 2017). Thus, short-term hypoxia could lead to a block in pre-mRNA splicing that is reminiscent of other stress responses, such as heat shock (Biamonti and Caceres 2009; Shalgi et al. 2014). During adaptation, this is then translated into a cell type-specific splicing program to enable continued cell survival under prolonged hypoxia.

In summary, the post-transcriptional response to hypoxia is a highly complex and multilayered process that superimposes and fine-tunes transcriptional regulation by HIF proteins. We took a step toward a deeper understanding of these processes and the crosstalk between HIFs and RBPs, which is necessary to open up new approaches in cancer therapy. To this end, we achieved a comparable data set of hypoxia-driven changes in RBP levels and alternative splicing in two unrelated cancer types. This 
approach enabled us to reveal a new layer in the regulation of hypoxia adaptation by MBNL2. We identified novel MBNL2 targets and demonstrated MBNL2 control of VEGFA expression at mRNA and protein level. This function in hypoxia adaptation is specific for MBNL2 and is not shared by its paralog MBNL1. Moreover, hypoxia regulation by MBNL2 seems to be universally functional in cancer cells. MBNL2, its targets and thus the complete MBNL2 signaling pathway have the potential to initialize new attempts in cancer research.

\section{MATERIALS AND METHODS}

\section{Cell culture and chronic hypoxia treatment}

A549 (DSMZ no. ACC-107) and MCF-7 cells (DSMZ no. ACC-115) were cultured in T75 flasks in DMEM (Sigma-Aldrich) or RPMI1640 medium (Sigma-Aldrich), respectively, supplemented with 10\% FBS (Thermo Fisher Scientific), $1 \mathrm{mM}$ sodium pyruvate (Thermo Fisher Scientific) and Penicillin Streptomycin (Pen Strep, Thermo Fisher Scientific).

For hypoxia treatment, $1 \times 10^{5}$ A549 cells or $2 \times 10^{5}$ MCF-7 cells were seeded in 12-well plates and incubated under normoxic conditions $\left(37^{\circ} \mathrm{C}, 5 \% \mathrm{CO}_{2}, 21 \% \mathrm{O}_{2}\right)$. Twenty-four hours after seeding, cells were exposed to hypoxia $\left(37^{\circ} \mathrm{C}, 5 \% \mathrm{CO}_{2}, 0.5 \%\right.$ $\mathrm{O}_{2}$, Eppendorf Galaxy $48 \mathrm{R}$ incubator). RNA samples were prepared $48 \mathrm{~h}$ later. Incubation of the cells under hypoxia $(0.5 \%$ oxygen) for $48 \mathrm{~h}$ mimics chronic hypoxia.

\section{Transfection}

For reverse transfection of siRNA, $1 \times 10^{5}$ A549 or $2 \times 10^{5}$ MCF-7 were transfected after the manufacturer's protocol using Lipofectamine RNAiMAX (Thermo Fisher Scientific). For subsequent RNA or protein isolation, cells were transfected in a 12-well format with 200 pmol of a nonsilencing control siRNA (siCTRL: 5'-UUCUCC GAACGUGUCACGU[dT][dT]-3'), an siRNA targeting MBNL2 (siMBNL2: 5'-CACCGUAACCGUUUGUAUG[dT][dT]-3') (Paul et al. 2006) or an siRNA targeting MBNL1 (siMBNL1: 5'-AAGGACGAGGTCATTAGCCAT[dT][dT]-3') (Qiagen). Samples were prepared or assays performed $48-72 \mathrm{~h}$ post-transfection.

\section{RNA isolation}

Total RNA from A549 and MCF-7 cells was isolated either using TRIzol reagent (Invitrogen), followed by TURBO DNase (Thermo Fisher Scientific) treatment or by using the miRNeasy Mini kit (Qiagen), including the optional on-column DNA digestion with the RNase-Free DNase Set (Qiagen). After isolation, $500 \mathrm{ng}$ RNA were quality checked on a $1 \%$ agarose gel.

\section{RT-PCR and RT-qPCR}

RT-PCR analysis and RT-qPCR quantification was performed as in (Weigand et al. 2012). Oligonucleotide sequences are listed in Supplemental Table 11. All PCR products were verified by sequencing.

\section{RNA-sequencing}

Total RNA from normoxic $\left(21 \% \mathrm{O}_{2}\right)$ and hypoxic $\left(48 \mathrm{~h}, 0.5 \% \mathrm{O}_{2}\right)$ A549 and MCF-7 cells ( $n=2)$ was used to generate rRNA-depleted (RiboZero, Illumina) strand-specific cDNA libraries (TruSeq stranded mRNA library preparation kit, Illumina). Libraries were prepared by the Core Unit Systems Medicine (University of Würzburg) according to the manufacturer's protocol omitting the poly $(A)$ enrichment step. All libraries were sequenced on a NextSeq 500 (Illumina), obtaining 75 nt single-end reads. Approximately 100 million reads were obtained for each library.

For the MBNL2 knockdown experiments $(n=2)$, total RNA from hypoxic ( $48 \mathrm{~h}, 0.5 \% \mathrm{O}_{2}$ ) MCF-7 cells transfected either with a nonsilencing control siRNA (siCTRL) or an siRNA targeting MBNL2 (siMBNL2) was used to generate poly(A)-enriched strand-specific cDNA libraries (mRNA selection, TruSeq stranded mRNA library preparation kit, Illumina). Libraries were prepared by the Core Unit Systems Medicine (University of Würzburg) according to the manufacturer's protocol. Libraries were sequenced on a NextSeq 500 (Illumina), obtaining 75 nt single-end reads (Core Unit Systems Medicine, University of Würzburg). Approximately 80 million reads were obtained for each library.

\section{RNA-seq data analyses}

Reads were mapped to the human genome (GRCh38/hg38 assembly) using the splice-aware aligner STAR version 2.4.5a (Dobin et al. 2013). The htseq-count script from the HTSeq python package version 0.6.1 (Anders et al. 2015) was used to count reads within genes annotated in GENCODE version 24. Differential expression analysis between hypoxic and normoxic conditions, as well as siMBNL2 and siCTRL, was performed using the R/Bioconductor package DESeq2 (Love et al. 2014). Differentially expressed genes were defined based on an adjusted $P<0.05$ and an absolute fold change $>1.5$. Alternative splicing events were detected using rMATS version 3.2.5 (Shen et al. 2014), with default parameters, considering only reads mapped to splice junctions. A cutoff was adopted at a false discovery rate $(\mathrm{FDR})<0.05$ and an absolute change in "percent spliced in" (| $\Delta \mathrm{PSI} \mid) \geq 10 \%$. Further, we required at least 10 and five reads (average over replicates) supporting the junctions of inclusion and skipping events, respectively, and an average inclusion level $\geq 10 \%$ in either one or the other condition, to minimize false positives. For both differentially expressed genes and alternative splicing events, we kept only genes with a minimum expression level of $>1$ transcript per million (TPM) in at least one of the four samples obtained for each cell type/condition. Gene Ontology (GO) enrichment analysis was performed using the enrichGO function in clusterProfiler package (version 3.6.0) (Yu et al. 2012) in $R$ (version 3.4.3) applying a cutoff of 0.05 for $P$-value and $Q$-value.

\section{Gene expression correlation}

Gene expression data from patients with breast invasive carcinoma (BRCA, 1088 patients) and lung adenocarcinoma (LUAD, 533 patients) of The Cancer Genome Atlas (TCGA) PanCancer Atlas (Hoadley et al. 2018) were downloaded from the Genomics Data Commons (GDC) Data Portal (https://portal.gdc.cancer .gov/) and cBioPortal (http://www.cbioportal.org/). In order to test for an association between MBNL2 mRNA levels and the 
expression of other genes, Pearson's product-moment correlation was calculated on $\log _{2}$-transformed fragments per kilobase of transcript per million mapped reads (FPKM) values. Hypoxia response genes were the 556 genes that were consistently induced in A549 and MCF-7 cells in response to hypoxia (Fig. 1D). Analyses were performed for all genes with a mean expression of FPKM > 1 over all samples per cohort, that is, 14,922 genes in BRCA and 15,590 genes in LUAD, including 455 and 469 hypoxia response genes, respectively. Using two different cutoffs, we detected 6814 genes with a significant Pearson correlation coefficient $(P C C)>0.15$ and 2174 genes with PCC $>0.3$ in the BRCA data set as well as 5433 genes with $P C C>0.15$ and 3150 genes with PCC $>0.3$ in the LUAD data set $\left(P<10 \times 10^{-6}\right.$, BenjaminiHochberg correction).

\section{Fluorescence detection and Western blot analysis}

For Western blot analyses, A549 and MCF-7 cells were lysed in lysis buffer [137 mM NaCl, 10\% glycerol, $20 \mathrm{mM}$ Tris- $\mathrm{HCl} \mathrm{pH}$ 8.0, 2 mM EDTA pH 8.0, 1\% Igepal, $5 \mu \mathrm{L}$ protease inhibitor cocktail (Sigma-Aldrich)] for $20 \mathrm{~min}$ on ice. After centrifugation (15 min at $\left.17,000 \mathrm{~g}, 4^{\circ} \mathrm{C}\right)$ the protein content of the samples was determined in three technical replicates according to the Bradford method. A total of $10-20 \mu \mathrm{g}$ protein extract was loaded onto $10 \%$ SDS polyacrylamide gels and blotted onto PVDF membranes (Bio-Rad). Before blotting, total lane protein (loading control) was visualized using 2,2,2-trichloroethanol (Ladner et al. 2004). Membranes were blocked with $2 \%$ skim milk. MBNL1 antibodies MB1a(4A8) (Holt et al. 2007) was a kind gift from Wolfson Centre for Inherited Neuromuscular Disease (CIND). Horseradish peroxidase-conjugated anti-mouse or anti-rabbit lgG (Jackson ImmunoResearch) were used as secondary antibodies. Blots were developed with the ECL system (Bio-Rad) or Amersham ECL select (GE Healthcare) for weaker signals. Images were detected using the ChemiDoc Imaging System (Bio-Rad). Membranes were reused for a maximum of three times.

For MBNL2 splicing assays, protein lysate was prepared with MEF $12 \mathrm{KO}$ cells harvested after $48 \mathrm{~h}$ post-transfection with 750 ng of GFP-MBNL1-42, GFP-MBNL2-40, or GFP expressing vector and 200 ng of VEGFA BS3 minigene. The cells were resuspended in 1× RIPA buffer supplemented with Halt Protease Inhibitor Cocktail (Thermo Fisher) and incubated on ice with vortexing at $10 \mathrm{~min}$ interval. The lysate was then incubated at $-80^{\circ} \mathrm{C} \mathrm{O} / \mathrm{N}$ followed by 20 min centrifugation at $4^{\circ} \mathrm{C}$ and $15,000 \mathrm{~g}$. The concentration of proteins was measured with BCA protein assay kit (Abcam). A total of $30 \mu \mathrm{g}$ of proteins was mixed with loading buffer but not denatured at high temperature and separated on $10 \%$ SDS polyacrylamide gels. GFP-MBNL fusion proteins and control GFP were detected at $488 \mathrm{~nm}$ excitation, protein marker was visualized at $635 \mathrm{~nm}$ excitation at Amersham Typhoon RGB laser scanner. Subsequently, the samples were transferred to a nitrocellulose membrane (Protran BA 85, Whatman) using a wet transfer apparatus $\left(1 \mathrm{~h}, 100 \mathrm{~V}, 4^{\circ} \mathrm{C}\right)$. The membrane was blocked for $1 \mathrm{~h}$ with $5 \%$ skim milk in PBST buffer [phosphate-buffered saline (PBS), 0.1\% Tween-20] and incubated with a primary antibody against $\alpha$-Tubulin (1:2000, Abcam, ab52866). Anti-rabbit (1:2500, Agrisera, AS09602) secondary antibody was conjugated with horseradish peroxidase and detected using Immobilon Forte Western HRP substrate (Millipore).

Antibodies are listed in Supplemental Table 12.

\section{VEGF-A detection by enzyme-linked immunosorbent assay (ELISA)}

Human VEGF-A ELISA Kit (Sigma-Aldrich) was used to quantify secreted human VEGF-A protein in cell culture supernatants from normoxic and hypoxic ( $48 \mathrm{~h}, 0.5 \% \mathrm{O}_{2}$ ) A549 and MCF-7 cells transfected either with a nonsilencing control siRNA (siCTRL) or an siRNA targeting MBNL2 (siMBNL2). Cell culture supernatants were diluted 1:2 in Sample Diluent Buffer B and protein detection was performed according to the manufacturer's protocol.

\section{Cell viability assay}

A549 and MCF-7 cells were transfected as described above and incubated under normoxic or hypoxic conditions $(48 \mathrm{~h}, 0.5 \%$ $\mathrm{O}_{2}$ ). Twenty-four hours later, cisplatin was added at final concentrations of 10 or $20 \mu \mathrm{M}$. After another $24 \mathrm{~h}$ under normoxia/hypoxia the cells were fixed with $0.5 \%$ formaldehyde in PBS and stained with $0.5 \%$ crystal violet in PBS. After three washing steps with PBS the cells were incubated with $33 \%$ acetic acid. Samples were transferred to a 96 well plate. Absorption at $570 \mathrm{~nm}$ was measured in a TECAN infinite M 200 Pro plate reader. Absorption was normalized to normoxic control cells with the respective cisplatin concentration.

\section{Transwell migration assay}

A549 cells were transfected as described above. $24 \mathrm{~h}$ later, cells were serum-starved (0\% FBS) and exposed to hypoxia (48 h, $0.5 \% \mathrm{O}_{2}$ ). Cells were washed in PBS and harvested using $40 \mu \mathrm{L}$ Trypsin-EDTA (0.05\% trypsin, $0.02 \%$ EDTA in PBS). The harvested cells were collected and resuspended in starvation medium. We used 24-well transwell chambers (Thincert cell culture inserts, pore diameter $8 \mu \mathrm{m}$, translucent PET membrane, Greiner). A total of $5 \times 10^{4}$ cells in $100 \mu \mathrm{L}$ starvation medium were seeded per insert, which was placed into a lower well containing $700 \mu \mathrm{L}$ DMEM medium supplemented with 10\% FBS (Thermo Fisher Scientific), $1 \mathrm{mM}$ sodium pyruvate (Thermo Fisher Scientific) and Pen Strep (Thermo Fisher Scientific) without or with $100 \mathrm{ng} / \mu \mathrm{L} \mathrm{hEGF}$ as a positive control or starvation medium ( $0 \% \mathrm{FBS}$ ) as a negative control. After $5 \mathrm{~h}$ under hypoxic conditions, the experiment was stopped. Cells from the insert were removed with a cotton swab. Migrated cells were washed with PBS and fixed with methanol. Migrated cells were stained with crystal violet (0.5\% in PBS) and the membranes were mounted using Pertex (Histolab). Quantification of migrated cells was done by counting six fields per well from random sites of the transwell. No cells were detectable in the negative control.

\section{mRNA decay assay}

For mRNA decay experiments, MCF-7 cells transfected with either a nonsilencing siRNA (siCTRL) or an siRNA targeting MBNL2 (siMBNL2) were incubated under hypoxic conditions (48 h, 0.5\% $\mathrm{O}_{2}$ ). After $48 \mathrm{~h}$ cells were treated with $5 \mu \mathrm{g} / \mathrm{mL}$ actinomycin D (Sigma) for $30 \mathrm{~min}$. Total RNA was extracted at the indicated time points and mRNA levels were quantified by RT-qPCR. MRNA levels were normalized to RPLPO mRNA levels and plotted against time. 


\section{Splicing minigene assays}

VEGFA splicing minigenes based on the ATP2A1 minigene were prepared as previously described (Taylor et al. 2018). Briefly, three sequences derived from the VEGFA $3^{\prime} U T R$, carrying potential MBNL binding sites were obtained in two PCR steps introducing Notl and Sall restriction sites.

Mouse Embryonic Fibroblasts (MEFs) with MBNL1 and MBNL2 double knockout (12KO) were a gift from Maury Swanson (Lee et al. 2013). The growth conditions of MEFs $12 \mathrm{KO}$ were previously described (Cywoniuk et al. 2017). Prior to transfection, the cells were plated in 12-well plates and transfected at 50\%-60\% confluence with Lipofectamine 3000 (Invitrogen) according to the manufacturer's protocol. Cotransfection was conducted with $200 \mathrm{ng}$ of a minigene and 750 ng of GFP-MBNL1-42, GFP-MBNL2-40, or GFP expression vectors. GFP-MBNL constructs were previously described (Sznajder et al. 2016; Konieczny et al. 2017). Cells were harvested $42 \mathrm{~h}$ after transfection.

Total RNA isolation was conducted using TRI reagent (SigmaAldrich) following the manufacturer's protocol. cDNA was synthesized using the SuperScript III Reverse Transcriptase kit (Invitrogen). Splicing analysis was performed with GoTaq DNA polymerase (Promega). Oligonucleotide sequences are listed in Supplemental Table 11.

\section{Preparation of radiolabeled RNA fragments}

The templates for transcription reactions for VEGFA RNA fragments derived from pre-mRNAs were obtained in PCR using specific oligonucleotide sets (TF/TR) having a promoter for polymerase T7. The PCR products were then purified prior to transcription reaction according to the manufacturer's protocol (A\&A Biotechnology). The transcription reaction and 5'-end radiolabeling were performed as previously described with slight changes (Sznajder et al. 2016). Briefly, transcription reaction was performed in $40 \mu \mathrm{L}$ composed of $10 \mu \mathrm{L}$ of DNA template, $1 \mathrm{mM}$ NTPs (Invitrogen), $3 \mathrm{mM}$ guanosine (Sigma-Aldrich), 20 U T7 RNA Polymerase (Promega), 1× T7 transcription buffer (Promega), 40 U Rnasin Plus RNase Inhibitor (Promega). Purification of transcript was conducted on a denaturing 6\% polyacrylamide gel (19:1 acrylamid:bisacrylamid) and through ethanol precipitation. For radiolabeling, 2 pmol of transcript was incubated with 2 pmol of $\left[\gamma_{-}{ }^{32} \mathrm{P}\right]$ ATP, $1 \cup$ Rnasin Plus, $10 \cup$ OptiKinase (Affymetrix), $1 \times$ reaction buffer (Affymetrix) and $\mathrm{dd}_{2} \mathrm{O}$ up to $10 \mu \mathrm{L}$, at $37^{\circ} \mathrm{C}$ for $30 \mathrm{~min}$. Labeled RNA was subsequently run on a denaturing $8 \%$ polyacrylamide gel (19:1) in 0.5 $\times$ TBE, at $100 \mathrm{~V}$ for $1 \mathrm{~h}$. A band of RNA was visualized on IP through FLA-5100 (FujiFilm), cut out followed by ethanol precipitation and resuspended in $20 \mu \mathrm{L} d d \mathrm{H}_{2} \mathrm{O}$. (CUG) 20 was a kind gift from Włodzimierz J. Krzyżosiak.

\section{Recombinant GST and (His) 6 -tagged MBNL1 and MBNL2}

GST fusions with MBNL1 and MBNL2 proteins encoded by exons 1-4 of the MBNL1 and MBNL2 with a (His) 6 -tag at the carboxyl terminus were prepared and purified as previously described (Sznajder et al. 2016). The protein concentration was measured using both Bradford assay and Sypro Ruby staining using 10\% SDS-PAGE.

\section{Quantification of MBNL-RNA interaction}

A biochemical assay based on double-membrane filtration analysis was performed as previously described with slight changes (Sznajder et al. 2016). Briefly, the reaction was performed in a $30 \mu \mathrm{L}$ volume. $5^{\prime}$-labeled transcripts $(0.05 \mathrm{nM})$ were first subjected to a denaturation-renaturation step. Subsequently, the RNAs were incubated with the indicated concentrations of MBNL1 and MBNL2 (ranging from 0 to $1250 \mathrm{nM}$ ) in buffer B containing $250 \mathrm{mM} \mathrm{NaCl}, 15 \mathrm{mM} \mathrm{KCl}, 50 \mathrm{mM}$, Tris- $\mathrm{HCl} \mathrm{pH}$ 8.0, $0.05 \%$ Tween-20, $1 \mathrm{mM} \mathrm{MgCl} 2$ at $37^{\circ} \mathrm{C}$ for $15 \mathrm{~min}$. An amount of $25 \mu \mathrm{L}$ samples were loaded onto a filter binding apparatus with nitrocellulose (Protran BA 85, Whatman) and nylon (Hybond $\mathrm{N}+$, Amersham) membranes previously wetted in buffer $\mathrm{B}$. The signal from membranes was visualized after IP through a FLA-5100 and quantified using the Multi Gauge software (FujiFilm). The $K_{D}$ values of the MBNL-RNA complexes were calculated based on the signal of free RNA and MBNL-RNA complexes from three experimental replicates with GraphPad Prism using the one site specific binding curve equation

$$
\mathrm{Y}=\mathrm{B}_{\max } * \mathrm{X} /\left(K_{\mathrm{D}}+\mathrm{X}\right) .
$$

\section{Statistical analysis}

All bar graphs are reported as mean values \pm standard deviation. Statistical analysis was done using Student's t-test, two-tailed, paired, or Fisher's exact test, one-tailed, as indicated. Pearson's product-moment correlation was used to estimate the relationships between variables shown in scatter plots by using the cor. test function in $\mathrm{R}$ (version 3.4.3). The comparative enrichment of hypoxia-regulated AS events was tested with a two-sample test for equality of proportions, by using the prop.test function in $\mathrm{R}$ (version 3.4.3).

\section{DATA DEPOSITION}

The RNA-seq data of A549, MCF-7, and siMBNL2 experiments have been deposited with the Gene Expression Omnibus database (GEO; www.ncbi.nlm.nih.gov/geo/) and are available under accession numbers GSE131378 and GSE136231.

\section{SUPPLEMENTAL MATERIAL}

Supplemental material is available for this article.

\section{ACKNOWLEDGMENTS}

We thank Professor Dr. Beatrix Suess for the helpful discussions and Britta Kluge for excellent technical assistance. We thank the Wolfson Centre for Inherited Neuromuscular Disease (CIND) for donating the MBNL1 antibody. This work was supported by the IZKF at the University of Würzburg (project Z-6). This work was supported by the Deutsche Forschungsgemeinschaft (SFB902/ B14 to J.E.W. and SFB902/B13 to Z.K.), the Dr. Hans Messer Stiftung (to J.E.W.), the Dr. Ing. Wilhelm und Maria KirmserStiftung (to S.F.), the Foundation for Polish Science, TEAM (POIR.04.04.00-00-5C0C/17-00 to K.S.) and the Polish National Science Centre (UMO-2017/24/C/NZ1/00112 to K.T.). 
Received September 17, 2019; accepted February 27, 2020.

\section{REFERENCES}

Adereth Y, Dammai V, Kose N, Li R, Hsu T. 2005. RNA-dependent integrin $\alpha 3$ protein localization regulated by the Muscleblind-like protein MLP1. Nat Cell Biol 7: 1240-1247. doi:10.1038/ncb1335

Anczuków O, Krainer AR. 2016. Splicing-factor alterations in cancers. RNA 22: 1285-1301. doi:10.1261/rna.057919.116

Anders S, Pyl PT, Huber W. 2015. HTSeq-a Python framework to work with high-throughput sequencing data. Bioinformatics 31: 166169. doi:10.1093/bioinformatics/btu638

Anufrieva KS, Shender VOC, Arapidi GP, Pavlyukov MS, Shakhparonov MI, Shnaider PV, Butenko IO, Lagarkova MA, Govorun VM. 2018. Therapy-induced stress response is associated with downregulation of pre-mRNA splicing in cancer cells. Genome Med 10: 49. doi:10.1186/s13073-018-0557-y

Arcondeguy T, Lacazette E, Millevoi S, Prats H, Touriol C. 2013. VEGFA mRNA processing, stability and translation: a paradigm for intricate regulation of gene expression at the post-transcriptional level. Nucleic Acids Res 41: 7997-8010. doi:10.1093/nar/gkt539

Arif A, Yao P, Terenzi F, Jia J, Ray PS, Fox PL. 2018. The GAIT translational control system. Wiley Interdiscip Rev RNA 9: e1441. doi:10 .1002/wrna.1441

Batra R, Charizanis K, Manchanda M, Mohan A, Li M, Finn DJ, Goodwin M, Zhang C, Sobczak K, Thornton CA, et al. 2014. Loss of MBNL leads to disruption of developmentally regulated alternative polyadenylation in RNA-mediated disease. Mol Cell 56: 311322. doi:10.1016/j.molcel.2014.08.027

Biamonti G, Caceres JF. 2009. Cellular stress and RNA splicing. Trends Biochem Sci 34: 146-153. doi:10.1016/j.tibs.2008.11.004

Blancher C, Moore JW, Talks KL, Houlbrook S, Harris AL. 2000. Relationship of hypoxia-inducible factor (HIF)- $1 \alpha$ and HIF-2 $\alpha$ expression to vascular endothelial growth factor induction and hypoxia survival in human breast cancer cell lines. Cancer Res 60: 7106-7113.

Bowler E, Porazinski S, Uzor S, Thibault P, Durand M, Lapointe E, Rouschop KMA, Hancock J, Wilson I, Ladomery M. 2018. Hypoxia leads to significant changes in alternative splicing and elevated expression of CLK splice factor kinases in PC3 prostate cancer cells. BMC Cancer 18: 355. doi:10.1186/s12885-0184227-7

Brady LK, Wang H, Radens CM, Bi Y, Radovich M, Maity A, Ivan C, Ivan M, Barash Y, Koumenis C. 2017. Transcriptome analysis of hypoxic cancer cells uncovers intron retention in EIF2B5 as a mechanism to inhibit translation. PLoS Biol 15: e2002623. doi:10 .1371/journal.pbio.2002623

Bray F, Ferlay J, Soerjomataram I, Siegel RL, Torre LA, Jemal A. 2018. Global cancer statistics 2018: GLOBOCAN estimates of incidence and mortality worldwide for 36 cancers in 185 countries. CA Cancer J Clin 68: 394-424. doi:10.3322/caac.21492

Carraway KR, Johnson EM, Kauffmann TC, Fry NJ, Mansfield KD. 2017. Hypoxia and hypoglycemia synergistically regulate mRNA stability. RNA Biol 14: 938-951. doi:10.1080/15476286.2017 .1311456

Cerami E, Gao J, Dogrusoz U, Gross BE, Sumer SO, Aksoy BA, Jacobsen A, Byrne CJ, Heuer ML, Larsson E, et al. 2012. The cBio Cancer Genomics Portal: an open platform for exploring multidimensional cancer genomics data. Cancer Discov 2: 401-404. doi:10.1158/2159-8290.CD-12-0095

Charizanis K, Lee KY, Batra R, Goodwin M, Zhang C, Yuan Y, Shiue L, Cline M, Scotti MM, Xia G, et al. 2012. Muscleblind-like 2-mediated alternative splicing in the developing brain and dysregulation in myotonic dystrophy. Neuron 75: 437-450. doi:10.1016/j.neuron .2012.05.029
Chee NT, Lohse I, Brothers SP. 2019. mRNA-to-protein translation in hypoxia. Mol Cancer 18: 49. doi:10.1186/s12943-019-0968-4

Chen A, Sceneay J, Gödde N, Kinwel T, Ham S, Thompson EW, Humbert PO, Möller A. 2018. Intermittent hypoxia induces a metastatic phenotype in breast cancer. Oncogene 37: 4214-4225. doi:10.1038/s41388-018-0259-3

Cywoniuk P, Taylor K, Sznajder LJ, Sobczak K. 2017. Hybrid splicing minigene and antisense oligonucleotides as efficient tools to determine functional protein/RNAnteractions. Sci Rep 7: 17587. doi:10.1038/s41598-017-17816-x

deLorimier E, Hinman MN, Copperman J, Datta K, Guenza M, Berglund JA. 2017. Pseudouridine modification inhibits muscleblind-like 1 (MBNL1) binding to CCUG repeats and minimally structured RNA through reduced RNA flexibility. J Biol Chem 292: 4350-4357. doi:10.1074/jbc.M116.770768

Dobin A, Davis CA, Schlesinger F, Drenkow J, Zaleski C, Jha S, Batut P, Chaisson M, Gingeras TR. 2013. STAR: ultrafast universal RNA-seq aligner. Bioinformatics 29: 15-21. doi:10.1093/bioinformatics/ bts635

Du H, Cline MS, Osborne RJ, Tuttle DL, Clark TA, Donohue JP, Hall MP, Shiue L, Swanson MS, Thornton CA, et al. 2010. Aberrant alternative splicing and extracellular matrix gene expression in mouse models of myotonic dystrophy. Nat Struct Mol Biol 17: 187-193. doi:10.1038/nsmb.1720

Escobar-Hoyos L, Knorr K, Abdel-Wahab O. 2019. Aberrant RNA splicing in cancer. Annu Rev Cancer Biol 3: 167-185. doi:10 .1146/annurev-cancerbio-030617-050407

Fardaei M, Rogers MT, Thorpe HM, Larkin K, Hamshere MG, Harper PS, Brook JD. 2002. Three proteins, MBNL, MBLL and $M B X L$, co-localize in vivo with nuclear foci of expanded-repeat transcripts in DM1 and DM2 cells. Hum Mol Genet 11: 805-814. doi:10.1093/hmg/11.7.805

Fish L, Pencheva N, Goodarzi H, Tran H, Yoshida M, Tavazoie SF. 2016. Muscleblind-like 1 suppresses breast cancer metastatic colonization and stabilizes metastasis suppressor transcripts. Genes Dev 30: 386-398. doi:10.1101/gad.270645.115

Forsythe JA, Jiang BH, lyer NV, Agani F, Leung SW, Koos RD, Semenza GL. 1996. Activation of vascular endothelial growth factor gene transcription by hypoxia-inducible factor 1 . Mol Cell Biol 16: 4604-4613. doi:10.1128/MCB.16.9.4604

Fry NJ, Law BA, Ilkayeva OR, Holley CL, Mansfield KD. 2017. N6 methyladenosine is required for the hypoxic stabilization of specific mRNAs. RNA 23: 1444-1455. doi:10.1261/rna.061044 117

Gallego-Paez LM, Bordone MC, Leote AC, Saraiva-Agostinho N, Ascensão-Ferreira M, Barbosa-Morais NL. 2017. Alternative splicing: the pledge, the turn, and the prestige: the key role of alternative splicing in human biological systems. Hum Genet 136: 10151042. doi:10.1007/s00439-017-1790-y

Gao J, Aksoy BA, Dogrusoz U, Dresdner G, Gross B, Sumer SO, Sun Y, Jacobsen A, Sinha R, Larsson E, et al. 2013. Integrative analysis of complex cancer genomics and clinical profiles using the cBioPortal. Sci Signal 6: pl1. doi:10.1126/scisignal.2004088

Gossage L, Eisen T, Maher ER. 2015. VHL, the story of a tumour suppressor gene. Nat Rev Cancer 15: 55-64. doi:10.1038/nrc3844

Graham K, Unger E. 2018. Overcoming tumor hypoxia as a barrier to radiotherapy, chemotherapy and immunotherapy in cancer treatment. Int J Nanomedicine 13: 6049-6058. doi:10.2147/IJN .S140462

Han J, Li J, Ho JC, Chia GS, Kato H, Jha S, Yang H, Poellinger L, Lee KL. 2017. Hypoxia is a key driver of alternative splicing in human breast cancer cells. Sci Rep 7: 4108. doi:10.1038/s41598017-04333-0

Hettiarachchi GK, Katneni UK, Hunt RC, Kames JM, Athey JC, Bar H, Sauna ZE, McGill JR, Ibla JC, Kimchi-Sarfaty C. 2019. Translational 
and transcriptional responses in human primary hepatocytes under hypoxia. Am J Physiol Gastrointest Liver Physiol 316: G720G734. doi:10.1152/ajpgi.00331.2018

Hoadley KA, Yau C, Hinoue T, Wolf DM, Lazar AJ, Drill E, Shen R, Taylor AM, Cherniack AD, Thorsson V, et al. 2018. Cell-of-origin patterns dominate the molecular classification of 10,000 tumors from 33 types of cancer. Cell 173: 291-304.e296. doi:10.1016/j .cell.2018.03.022

Holt I, Mittal S, Furling D, Butler-Browne GS, Brook JD, Morris GE. 2007. Defective mRNA in myotonic dystrophy accumulates at the periphery of nuclear splicing speckles. Genes Cells 12: 1035-1048. doi:10.1111/j.1365-2443.2007.01112.x

Hu X, Wu R, Shehadeh LA, Zhou Q, Jiang C, Huang X, Zhang L, Gao F, Liu X, Yu H, et al. 2014. Severe hypoxia exerts parallel and cell-specific regulation of gene expression and alternative splicing in human mesenchymal stem cells. BMC Genomics 15: 303. doi:10 .1186/1471-2164-15-303

Jafarifar F, Yao P, Eswarappa SM, Fox PL. 2011. Repression of VEGFA by CA-rich element-binding microRNAs is modulated by hnRNP L. EMBO J 30: 1324-1334. doi:10.1038/emboj.2011.38

Jakubauskiene E, Vilys L, Makino Y, Poellinger L, Kanopka A. 2015. Increased serine-arginine (SR) protein phosphorylation changes pre-mRNA splicing in hypoxia. J Biol Chem 290: 18079-18089. doi:10.1074/jbc.M115.639690

Kemmerer K, Fischer S, Weigand JE. 2018. Auto- and cross-regulation of the hnRNPs D and DL. RNA 24: 324-331. doi:10.1261/rna 063420.117

Khabar KS. 2017. Hallmarks of cancer and AU-rich elements. Wiley Interdiscip Rev RNA 8: e1368. doi:10.1002/wrna.1368

Konieczny P, Stepniak-Konieczna E, Sobczak K. 2014. MBNL proteins and their target RNAs, interaction and splicing regulation. Nucleic Acids Res 42: 10873-10887. doi:10.1093/nar/gku767

Konieczny P, Stepniak-Konieczna E, Taylor K, Sznajder LJ, Sobczak K. 2017. Autoregulation of MBNL1 function by exon 1 exclusion from MBNL1 transcript. Nucleic Acids Res 45: 1760-1775. doi:10.1093/ nar/gkw1158

Konieczny P, Stepniak-Konieczna E, Sobczak K. 2018. MBNL expression in autoregulatory feedback loops. RNA Biol 15: 1-8. doi:10 .1080/15476286.2017.1384119

Ladner CL, Yang J, Turner RJ, Edwards RA. 2004. Visible fluorescent detection of proteins in polyacrylamide gels without staining Anal Biochem 326: 13-20. doi:10.1016/j.ab.2003.10.047

Lambert N, Robertson A, Jangi M, McGeary S, Sharp PA, Burge CB. 2014. RNA Bind-n-Seq: quantitative assessment of the sequence and structural binding specificity of RNA binding proteins. Mol Cell 54: 887-900. doi:10.1016/j.molcel.2014.04.016

Lee KY, Li M, Manchanda M, Batra R, Charizanis K, Mohan A, Warren SA, Chamberlain CM, Finn D, Hong H, et al. 2013 Compound loss of muscleblind-like function in myotonic dystrophy. EMBO Mol Med 5: 1887-1900. doi:10.1002/emmm .201303275

Lee YH, Jhuang YL, Chen YL, Jeng YM, Yuan RH. 2016. Paradoxical overexpression of MBNL2 in hepatocellular carcinoma inhibits tumor growth and invasion. Oncotarget 7: 65589-65601. doi:10 18632/oncotarget.11577

Love MI, Huber W, Anders S. 2014. Moderated estimation of fold change and dispersion for RNA-seq data with DESeq2. Genome Biol 15: 550. doi:10.1186/s13059-014-0550-8

Masuda A, Andersen HS, Doktor TK, Okamoto T, Ito $M$, Andresen BS, Ohno K. 2012. CUGBP1 and MBNL1 preferentially bind to 3' UTRs and facilitate mRNA decay. Sci Rep 2: 209. doi:10.1038/srep00209

Meléndez-Rodríguez F, Roche O, Sanchez-Prieto R, Aragones J. 2018. Hypoxia-inducible factor 2-dependent pathways driving Von
Hippel-Lindau-deficient renal cancer. Front Oncol 8: 214. doi:10 3389/fonc. 2018.00214

Oltean S, Bates DO. 2014. Hallmarks of alternative splicing in cancer. Oncogene 33: 5311-5318. doi:10.1038/onc.2013.533

Paul S, Dansithong W, Kim D, Rossi J, Webster NJ, Comai L, Reddy S. 2006. Interaction of muscleblind, CUG-BP1 and hnRNP H proteins in DM1-associated aberrant IR splicing. EMBO J 25: 4271-4283. doi:10.1038/sj.emboj.7601296

Pereira B, Billaud M, Almeida R. 2017. RNA-binding proteins in cancer: old players and new actors. Trends Cancer 3: 506-528. doi:10.1016/j.trecan.2017.05.003

Perron G, Jandaghi P, Solanki S, Safisamghabadi M, Storoz C, Karimzadeh M, Papadakis Al, Arseneault M, Scelo G, Banks RE, et al. 2018. A general framework for interrogation of mRNA stability programs identifies RNA-binding proteins that govern cancer transcriptomes. Cell Rep 23: 1639-1650. doi:10.1016/j.celrep .2018 .04 .031

Rau F, Freyermuth F, Fugier C, Villemin JP, Fischer MC, Jost B, Dembele D, Gourdon G, Nicole A, Duboc D, et al. 2011. Misregulation of miR-1 processing is associated with heart defects in myotonic dystrophy. Nat Struct Mol Biol 18: 840-845. doi:10 .1038/nsmb.2067

Ray D, Kazan H, Cook KB, Weirauch MT, Najafabadi HS, Li X, Gueroussov S, Albu M, Zheng H, Yang A, et al. 2013. A compendium of RNA-binding motifs for decoding gene regulation. Nature 499: 172-177. doi:10.1038/nature12311

Robichaud N, Sonenberg N, Ruggero D, Schneider RJ. 2019. Translational control in cancer. Cold Spring Harb Perspect Biol 11: a032896. doi:10.1101/cshperspect.a032896

Samanta D, Semenza GL. 2018. Metabolic adaptation of cancer and immune cells mediated by hypoxia-inducible factors. Biochim Biophys Acta Rev Cancer 1870: 15-22. doi:10.1016/j.bbcan 2018.07.002

Schito L, Semenza GL. 2016. Hypoxia-inducible factors: master regulators of cancer progression. Trends Cancer 2: 758-770. doi:10 .1016/j.trecan.2016.10.016

Seiler M, Peng S, Agrawal AA, Palacino J, Teng T, Zhu P, Smith PG, Cancer Genome Atlas Research Network, Buonamici S, Yu L. 2018. Somatic mutational landscape of splicing factor genes and their functional consequences across 33 cancer types. Cell Rep 23: 282-296.e284. doi:10.1016/j.celrep.2018.01.088

Shalgi R, Hurt JA, Lindquist S, Burge CB. 2014. Widespread inhibition of posttranscriptional splicing shapes the cellular transcriptome following heat shock. Cell Rep 7: 1362-1370. doi:10.1016/j .celrep.2014.04.044

Shen S, Park JW, Lu ZX, Lin L, Henry MD, Wu YN, Zhou Q, Xing Y. 2014. rMATS: robust and flexible detection of differential alternative splicing from replicate RNA-seq data. Proc Natl Acad Sci 111: E5593-E5601. doi:10.1073/pnas.1419161111

Siegel RL, Miller KD, Jemal A. 2019. Cancer statistics, 2019. CA Cancer J Clin 69: 7-34. doi:10.3322/caac.21551

Sznajder ŁJ, Michalak M, Taylor K, Cywoniuk P, Kabza M, WojtkowiakSzlachcic A, Matłoka M, Konieczny P, Sobczak K. 2016. Mechanistic determinants of MBNL activity. Nucleic Acids Res 44: 10326-10342. doi:10.1093/nar/gkw915

Tabaglio T, Low DH, Teo WKL, Goy PA, Cywoniuk P, Wollmann H, Ho J, Tan D, Aw J, Pavesi A, et al. 2018. MBNL1 alternative splicing isoforms play opposing roles in cancer. Life Sci Alliance 1: e201800157. doi:10.26508/lsa.201800157

Tang R, Qi Q, Wu R, Zhou X, Wu D, Zhou H, Mao Y, Li R, Liu C, Wang L, et al. 2015. The polymorphic terminal-loop of pre-miR-1307 binding with MBNL1 contributes to colorectal carcinogenesis via interference with Dicer1 recruitment. Carcinogenesis 36: 867-875. doi:10.1093/carcin/bgv066 
Tang L, Zhao P, Kong D. 2019. Muscleblindlike 1 destabilizes Snail mRNA and suppresses the metastasis of colorectal cancer cells via the Snail/Ecadherin axis. Int J Oncol 54: 955-965.

Taylor K, Sznajder LJ, Cywoniuk P, Thomas JD, Swanson MS, Sobczak K. 2018. MBNL splicing activity depends on RNA binding site structural context. Nucleic Acids Res 46: 9119-9133. doi:10 $.1093 /$ nar/gky565

Teye EK, Sido A, Xin P, Finnberg NK, Gokare P, Kawasawa YI, Salzberg AC, Shimko S, Bayerl M, Ehmann WC, et al. 2017. PIGN gene expression aberration is associated with genomic instability and leukemic progression in acute myeloid leukemia with myelodysplastic features. Oncotarget 8: 29887-29905. doi:10.18632/oncotarget.15136

Vaupel P, Mayer A, Höckel M. 2004. Tumor hypoxia and malignant progression. Methods Enzymol 381: 335-354. doi:10.1016/ S0076-6879(04)81023-1

Vaupel P, Höckel M, Mayer A. 2007. Detection and characterization of tumor hypoxia using $\mathrm{pO}_{2}$ histography. Antioxid Redox Signal 9: 1221-1235. doi:10.1089/ars.2007.1628

Wang ET, Cody NA, Jog S, Biancolella M, Wang TT, Treacy DJ, Luo S, Schroth GP, Housman DE, Reddy S, et al. 2012. Transcriptomewide regulation of pre-mRNA splicing and mRNA localization by muscleblind proteins. Cell 150: 710-724. doi:10.1016/j.cell.2012 .06 .041
Weigand JE, Boeckel JN, Gellert P, Dimmeler S. 2012. Hypoxia-induced alternative splicing in endothelial cells. PLoS One 7: e42697. doi:10.1371/journal.pone.0042697

Wilson WR, Hay MP. 2011. Targeting hypoxia in cancer therapy. Nat Rev Cancer 11: 393-410. doi:10.1038/nrc3064

Yancopoulos GD. 2010. Clinical application of therapies targeting VEGF. Cell 143: 13-16. doi:10.1016/j.cell.2010.09.028

Yao P, Potdar AA, Ray PS, Eswarappa SM, Flagg AC, Willard B, Fox PL. 2013. The HILDA complex coordinates a conditional switch in the 3 '-untranslated region of the VEGFA mRNA. PLoS Biol 11: e1001635. doi:10.1371/journal.pbio.1001635

Yu G, Wang LG, Han Y, He QY. 2012. clusterProfiler: an R package for comparing biological themes among gene clusters. OMICS 16: 284-287. doi:10.1089/omi.2011.0118

Yuan JH, Liu XN, Wang TT, Pan W, Tao QF, Zhou WP, Wang F, Sun SH. 2017. The MBNL3 splicing factor promotes hepatocellular carcinoma by increasing PXN expression through the alternative splicing of IncRNA-PXN-AS1. Nat Cell Biol 19: 820-832. doi:10.1038/ ncb3538

Zhang J, Zheng Z, Wu M, Zhang L, Wang J, Fu W, Xu N, Zhao Z, Lao Y, Xu H. 2019. The natural compound neobractatin inhibits tumor metastasis by upregulating the RNA-binding-protein MBNL2. Cell Death Dis 10: 554. doi:10.1038/s41419-0191789-5 

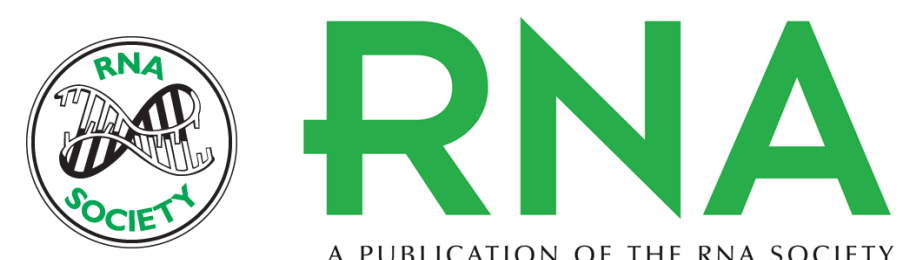

A PUBLICATION OF THE RNA SOCIETY

\section{Muscleblind-like 2 controls the hypoxia response of cancer cells}

Sandra Fischer, Antonella Di Liddo, Katarzyna Taylor, et al.

RNA 2020 26: 648-663 originally published online March 3, 2020

Access the most recent version at doi:10.1261/rna.073353.119

Supplemental Material

References

Creative Commons License

Email Alerting Service
http://rnajournal.cshlp.org/content/suppl/2020/03/03/rna.073353.119.DC1

This article cites 78 articles, 14 of which can be accessed free at: http://rnajournal.cshlp.org/content/26/5/648.full.html\#ref-list-1

This article is distributed exclusively by the RNA Society for the first 12 months after the full-issue publication date (see http://rnajournal.cshlp.org/site/misc/terms.xhtml). After 12 months, it is available under a Creative Commons License (Attribution-NonCommercial 4.0 International), as described at http://creativecommons.org/licenses/by-nc/4.0/.

Receive free email alerts when new articles cite this article - sign up in the box at the top right corner of the article or click here. 